\title{
Total Synthesis of Bacterial 5-(3-Indolyl)oxazole Alkaloids: Pimprinols A-C
}

\author{
Tímea Szabó (i) \\ Róbert Kormány \\ András Dancsó \\ Balázs Volk \\ Mátyás Milen* (1)
}

Egis Pharmaceuticals Plc., Directorate of Drug Substance Development, P. O. Box 100, 1475 Budapest, Hungary milen.matyas@egis.hu

This paper is dedicated to the memory of Professor József Reiter.
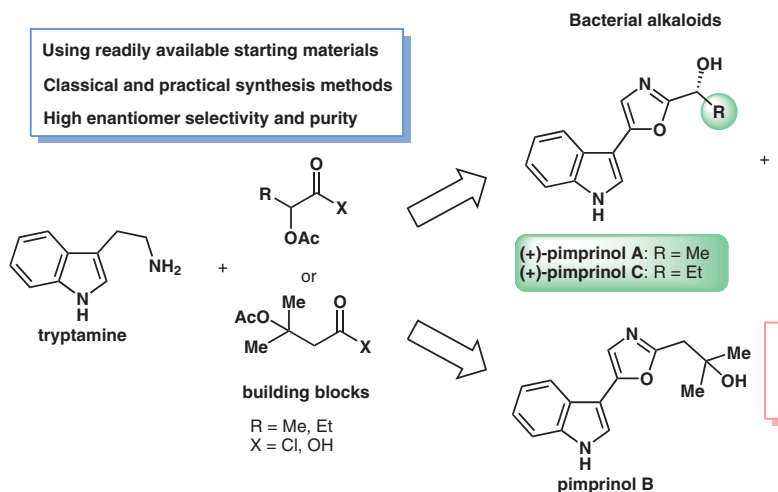

Antipodes of natural
products

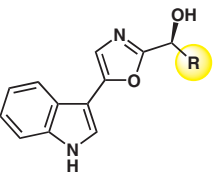
$(-)$-pimprinol $A: R=M e$
$(-)$-pimprinol $C: R=E t$

New synthesis of pimprinol A First total synthesis of pimprinol B and C

\begin{abstract}
Received: 16.10 .2019
Accepted after revision: 05.11.2019

Published online: 28.11 .2019

DOI: 10.1055/s-0039-1690336; Art ID: so-2019-d0030-op

License terms: (c) $(9$

( ) 2019. The Author(s). This is an open access article published by Thieme under the terms of the Creative Commons Attribution-NonDerivative-NonCommercial-License, permitting copying and reproduction so long as the original work is given appropriate credit. Contents may not be used for commercial purposes or adapted, remixed, transformed or built upon. (https://creativecommons.org/licenses/by-nc-nd/4.0/)

Abstract Pimprinols A, B, and C are bacterial 5-(3-indolyl)oxazole alkaloids that have been isolated from Streptomyces sp. Lv3-13. In this paper, we report a new synthesis of pimprinol $A$ and the first total synthesis of pimprinol B and pimprinol C. In addition, antipodes of the naturally occurring pimprinols $A$ and $C$, as well as the racemates of these two alkaloids were also prepared. In the pivotal step, the 1,3-oxazole ring was constructed by a Nicolaou's modified Robinson-Gabriel cyclization.
\end{abstract}

Key words oxazole, natural products, bacterial alkaloids, pimprinols A-C, total synthesis

Oxazole ring-containing compounds are significant representatives of natural products and synthetic pharmaceuticals. $^{[1-5]}$ The bacterial alkaloids pimprinols A-C $[(R)-\mathbf{1}, \mathbf{2}$, $(R)$-3; Figure 1], which belong to the 5-(3-indolyl)oxazole family, were isolated from the rare actinomycetes, Streptomyces sp. Lv3-13 by Müller and co-workers in 2012. ${ }^{6}$ All three extracted alkaloids $[(R)-\mathbf{1}, \mathbf{2},(R)-\mathbf{3}]$ were described as yellow oils and their structures were elucidated by UV, 1D and 2D NMR spectroscopy, and by HRMS (ESI, + ) analysis. The absolute configurations of pimprinol $\mathrm{A}[(R)-\mathbf{1}]$ and $\mathrm{C}$ $[(R)-3]$ were determined by Mosher ester analysis.

The only known synthesis of pimprinol A $[(R)-\mathbf{1}]$ was described in 2014 by Wu et al. (Scheme 1). ${ }^{7}$ In this one-pot method, compound $(R)-\mathbf{1}$ was prepared by treatment of 1 ( $1 \mathrm{H}$-indol-3-yl)ethanone (4) with L-threonine (5; 2 equiv) in the presence of $\mathrm{I}_{2}$ in DMSO at $110^{\circ} \mathrm{C}$. The product was

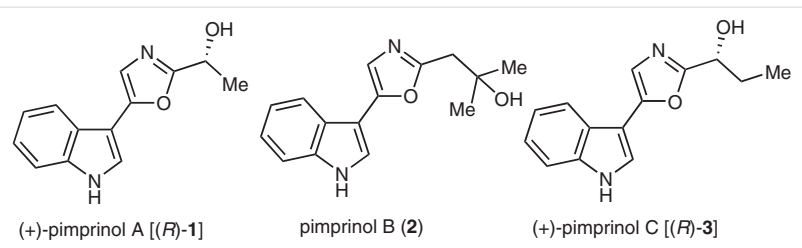

Figure 1 Chemical structures of pimprinols $A-C$

obtained in 70\% yield and $96 \%$ enantiomeric excess as a pale-brown solid. The alkaloids pimprinol B $(2)$ and C [(R)3] have not yet been synthesized.

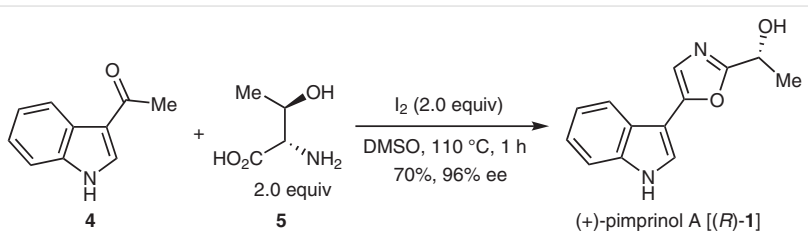

Scheme 1 Wu's synthesis of natural (+)-pimprinol A

Herein, we would like to report a new total synthesis of pimprinol $\mathrm{A}[(R)-\mathbf{1}]$ and the first total synthesis of pimprinol B $(2)$ and C [ $R)-3]$. In addition, antipodes and racemates of pimprinol $A$ and $C$ were also prepared.

Initially, the synthesis of racemic pimprinol A [(rac)-1] was investigated starting from the easily available DL-alanine [ $\mathrm{rac}$ )-6; Scheme 2]. 2-Chloro-1-methyl-2-oxoethyl acetate [ $\mathrm{rac})-7]$ was prepared as previously reported. $.^{8-10} \mathrm{Re}-$ action of $(\mathrm{rac})-\mathbf{6}$ with $\mathrm{NaNO}_{2}$ in glacial acetic acid, followed by treatment with an excess of $\mathrm{SOCl}_{2}$, afforded compound (rac)-7. Intermediate (rac)-9 was obtained by acylation of tryptamine (8) with (rac)-7 in the presence of $\mathrm{Et}_{3} \mathrm{~N}$ in $88 \%$ yield. Oxidation of ( $\mathrm{rac}$ )-9 with 2,3-dichloro-5,6-dicyano1,4-benzoquinone (DDQ) in a THF-water solvent mixture at $0{ }^{\circ} \mathrm{C}$ led to the corresponding acylamino-ketone ( $\left.\mathrm{rac}\right)-\mathbf{1 0}$ in 
excellent yield. The Robinson-Gabriel cyclization of acylamino-ketone (rac)-10 with propylphosponic anhydride $\left(\mathrm{T}^{\mathrm{P}}{ }^{\circledR}\right)$ under microwave conditions ${ }^{11}$ was unsuccessful, with the starting material being decomposed. Subsequently, the ring-closure reaction was attempted using Nicolaou's protocol with $\mathrm{POCl}_{3}$ in pyridine at room temperature. ${ }^{12}$ In this reaction, the desired 1,3-oxazole derivative ( $r a c)-11$ was obtained in $80 \%$ yield. Removal of the acetyl group by alkaline hydrolysis furnished the target molecule, racemic pimprinol A [(rac)-1], in almost quantitative yield.

By following the above synthetic route, natural (+)-pimprinol A $[(R)-\mathbf{1}]$ and its enantiomer (-)-pimprinol A $[(S)-\mathbf{1}]$ were prepared starting from $(R)$-alanine $[(R)$-6] and $(S)$-alanine $[(S)-6]$, respectively. It is important to note that the substitution of the amino group in alanine occurs by double inversion. ${ }^{10,13}$ Natural (+)-pimprinol A $[(R)-\mathbf{1}]$ was obtained in four steps from known (1R)-2-chloro-1-methyl-2oxoethyl acetate $[(R)-7]$ in $60 \%$ overall yield and in $98 \%$ ee. (-)-Pimprinol A [(S)-1] was synthesized from the corresponding acid chloride $(S)-7$ in a similar overall yield (61\%). The enantiomeric purity was $98 \%$ also in the final product, which was determined by chiral chromatographic separation on a polysaccharide stationary phase (column: $150 \times 4.6 \mathrm{~mm}$ Lux $5 \mu \mathrm{m}$ amylose- 1 , temperature: $20^{\circ} \mathrm{C}$, mobile phase: acetonitrile with $0.1 \%$ ethanolamine).

Racemic pimprinol C [(rac)-3] was prepared from the known 1-(chlorocarbonyl)propyl acetate $[(\mathrm{rac})-\mathbf{1 3}]^{8,14}$ by using an analogous procedure in four steps in $88 \%$ overall yield (Scheme 2). The natural alkaloid pimprinol C $[(R)-3]$ and its enantiomer $[(S)-3]$ were synthesized in a similar manner starting from $(1 R)-[(R)-\mathbf{1 3}]^{15}$ and $(1 S)$ - 1 -(chlorocarbonyl)propyl acetate $[(S)-\mathbf{1 3}],{ }^{16}$ respectively. The latter compounds were obtained in more than $80 \%$ yield and in $98 \%$ ee.
Next, the synthesis of pimprinol B (2) was studied from two readily available oxazole derivatives; methyl [5-( $1 \mathrm{H}$-indol-3-yl)-1,3-oxazol-2-yl] acetate (21) and the natural product labradorin 5 (22; Scheme 3). First, tryptamine (8) was converted into compounds $\mathbf{2 1}$ and $\mathbf{2 2}$ through a threestep procedure involving acylation with the appropriate acyl chlorides, DDQ mediated oxidation, and $\mathrm{T}^{\mathrm{P}} \mathrm{P}^{\circledR}$-promoted Robinson-Gabriel cyclodehydration under microwave conditions in a vent-and-reseal vessel. The reaction of ester 21 with MeMgI or MeLi was unsuccessful. In both cases the starting material was recovered. It is hypothesized that the reaction of oxazole $\mathbf{2 1}$ with Grignard or organolithium reagent produced an inactive dianion by deprotonation of both the indole $\mathrm{NH}$ and the active methylene group. Water addition to, or epoxidation of, the isopropylidene double bond in labradorin 5 (22) was unsuccessful with various reagents, such as $\mathrm{H}_{2} \mathrm{SO}_{4} / \mathrm{H}_{2} \mathrm{O}$ at room temperature, ${ }^{17}$ polyphosphoric acid (PPA) at $90{ }^{\circ} \mathrm{C},{ }^{18} \mathrm{Hg}(\mathrm{OAC})_{2} / \mathrm{THF}-\mathrm{H}_{2} \mathrm{O}$ then $\mathrm{NaOH} / \mathrm{NaBH}_{4},{ }^{19} \mathrm{HCl} / \mathrm{THF}-\mathrm{H}_{2} \mathrm{O}$ at reflux, ${ }^{20}$ and meta-chloroperoxybenzoic acid (mCPBA) at $0{ }^{\circ} \mathrm{C}^{21}$ Finally, upon treatment of labradorin 5 (22) with $50 \%$ aqueous $\mathrm{H}_{2} \mathrm{SO}_{4}$ in boiling 1,4-dioxane ${ }^{22}$ for 4 days, pimprinol B (2) was produced with $15 \%$ conversion and in $11 \%$ isolated yield. The main component of the residue was the starting material, with some decomposition detected. It is assumed that the poor reactivity of the isopropylidene double bond in compound $\mathbf{2 2}$ is caused by extensive conjugation.

Finally, a more efficient total synthesis of pimprinol B (2) was elaborated starting from commercially available 3hydroxy-3-methylbutanoic acid (23; Scheme 4). Protection of the tertiary hydroxyl group of $\mathbf{2 3}$ with acetyl chloride afforded carboxylic acid $\mathbf{2 4} .^{23}$ The latter intermediate and tryptamine (8) were coupled according to Methods A and B. First carbonyldiimidazole (CDI) as coupling agent was used
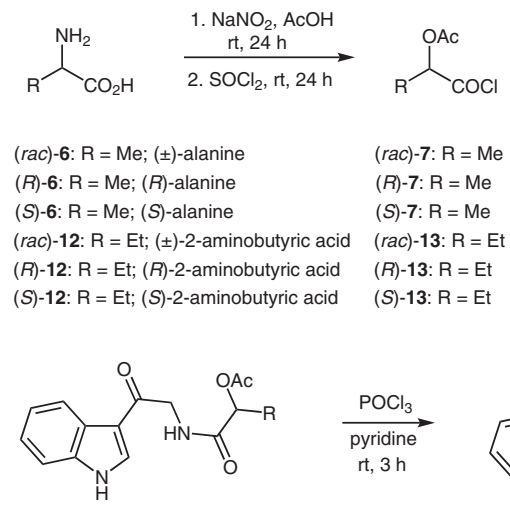

(rac)-10: $\mathrm{R}=\mathrm{Me} ; 95 \%$

(R)-10: $\mathrm{R}=\mathrm{Me} ; 91 \%$

(S)-10: $\mathrm{R}=\mathrm{Me} ; 93 \%$

(rac)-15: R = Et; $99 \%$

(R)-15: $\mathrm{R}=\mathrm{Et} ; 93 \%$

(S)-15: R = Et; 99\%

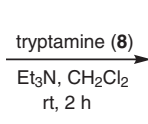

$\mathrm{t}, 2 \mathrm{~h}$

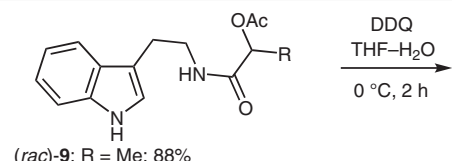

(R) $-9: \mathrm{R}=\mathrm{Me} ; 91 \%$

(S)-9: R = Me; $99 \%$

(rac)-14: R = Et; $99 \%$

(R)-14: $\mathrm{R}=\mathrm{Et} ; 99 \%$

(S)-14: R = Et; 99\%

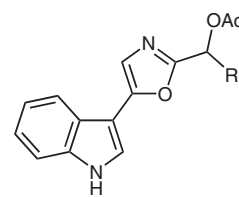

(rac)-11: $\mathrm{R}=\mathrm{Me} ; 80 \%$

(R)-11: $\mathrm{R}=\mathrm{Me} ; 77 \%$

(S)-11: R = Me; $74 \%$

(rac)-16: R = Et; $95 \%$

(R) $-16: \mathrm{R}=\mathrm{Et} ; 96 \%$

(S)-16: R $=\mathrm{Et}$; $92 \%$

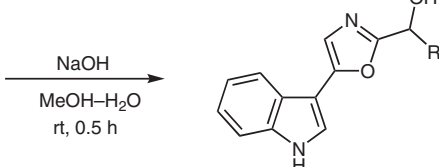

(rac)-1: R = Me; $( \pm$ )-pimprinol A; $97 \%$ (R)-1: $\mathrm{R}=\mathrm{Me}$; (+)-pimprinol A; $94 \%$ (S)-1: R = Me; (-)-pimprinol A; $89 \%$ (rac)-3: R = Et; ( \pm )-pimprinol C; $95 \%$

(R)-3: $\mathrm{R}=\mathrm{Et} ;(+)$-pimprinol C; $93 \%$

(S)-3: R = Et; (-)-pimprinol C; $96 \%$

Scheme 2 Synthesis of racemic and optically active pimprinol A and C 
<smiles>NCCc1c[nH]c2ccccc12</smiles>

8

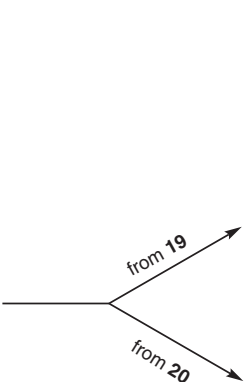<smiles>CNC(=O)NCCc1c[nH]c2ccccc12</smiles>

$$
\text { 17: } \mathrm{R}=\mathrm{CH}_{2} \mathrm{CO}_{2} \mathrm{Me} ; 62 \%
$$
18: $\mathrm{R}=\mathrm{CH}=\mathrm{CMe}_{2} ; 99 \%$<smiles>CC(=O)Cc1ncc(-c2c[nH]c3ccccc23)o1</smiles><smiles>CC(C)=Cc1ncc(-c2c[nH]c3ccccc23)o1</smiles>

22

labradorin 5

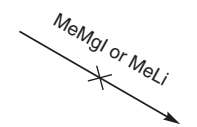

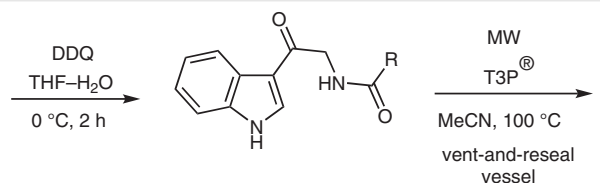

19: $\mathrm{R}=\mathrm{CH}_{2} \mathrm{CO}_{2} \mathrm{Me} ; 99 \%$ 20: $\mathrm{R}=\mathrm{CH}=\mathrm{CMe}_{2} ; 99 \%$
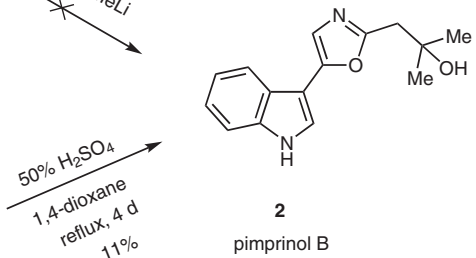

pimprinol B

Scheme 3 Attempts at synthesis of pimprinol B (2)

in the amide formation but this resulted in the required product in only moderate yield. However, applying 1[bis(dimethylamino)-methylene]-1H-1,2,3-triazolo[4,5b]pyridinium 3-oxide hexafluorophosphate (HATU) and Hünig's base, furnished the corresponding amide $\mathbf{2 5}$ in good yield. Although Method B resulted in a higher yield, the final product was contaminated with HATU, which was difficult to eliminate. Subsequent oxidation of intermediate 25 with DDQ in a THF-water solvent mixture provided $\beta$ keto amide 26. Reaction of compound $\mathbf{2 6}$ with $\mathrm{POCl}_{3}$ in pyridine, followed by alkaline hydrolysis of the ester group generated pimprinol B in 17\% (Method A) and 30\% (Method B) overall yields.
Pimprinols A-C were obtained as colorless crystals with sharp melting points, unlike the isolated samples. ${ }^{6}$ The analytical data of these synthesized alkaloids are in agreement with those reported for the natural products. The only significant difference was found in the specific rotation values. ${ }^{6}$

In conclusion, a convergent synthesis of racemic and optically active pimprinols $A$ and $C$ has been developed from readily available amino acids in six steps using practical and convenient synthetic methodology. Furthermore, the first total synthesis of optically inactive pimprinol B was accomplished in five steps starting from commercially available 3-hydroxy-3-methylbutanoic acid. In addition, 25 new

\footnotetext{
Scheme 4 First total synthesis of pimprinol B
} 
compounds were also synthesized as intermediates during the preparations. In each case, the modified RobinsonGabriel cyclization was employed as the key step.

Reactions under microwave conditions were carried out with a MicroSYNTH T640 in 'vent-and-reseal' vessels with an ATC-FO automatic temperature control and limitation of maximum power to 200$300 \mathrm{~W}$. All melting points were determined with a Büchi B-540 capillary melting point apparatus and are uncorrected. IR spectra were obtained with a Bruker Alpha FT-IR spectrophotometer in $\mathrm{KBr}$ pellets or film. ${ }^{1} \mathrm{H}$ and ${ }^{13} \mathrm{C}$ NMR spectra were recorded in DMSO- $d_{6}, \mathrm{CDCl}_{3}$ or $\mathrm{CD}_{3} \mathrm{OD}$ in $5 \mathrm{~mm}$ tubes at r.t., with a Bruker Avance III HD 600 (600 and $150 \mathrm{MHz}$ for ${ }^{1} \mathrm{H}$ and ${ }^{13} \mathrm{C}$ NMR spectra, respectively), or a Bruker Avance III 400 ( 400 and $100 \mathrm{MHz}$ for ${ }^{1} \mathrm{H}$ and ${ }^{13} \mathrm{C}$ NMR spectra, respectively) spectrometer with the deuterium signal of the solvent as the lock and TMS as the internal standard. Chemical shifts $(\delta)$ and coupling constants $(J)$ are given in ppm and in $\mathrm{Hz}$, respectively. The following abbreviations were used to designate multiplicities: $s=$ singlet, $\mathrm{d}=$ doublet, $\mathrm{t}=$ triplet, $\mathrm{q}=$ quartet, $\mathrm{sp}=$ septet, $\mathrm{m}=$ multiplet, $\mathrm{br}=$ broad. Mass spectra were recorded with a Bruker O-TOF MAXIS Impact mass spectrometer coupled to a Dionex Ultimate 3000 RS HPLC system with a diode array detector. Reactions were monitored by thin-layer chromatography (TLC) carried out on silica gel plates (60 $\mathrm{F}_{254}$ ) using UV light as visualizing agent. Purifications by flash column chromatography were carried out using Merck 107736 silica gel $60 \mathrm{H}$ using a hexane- $\mathrm{CH}_{2} \mathrm{Cl}_{2}$ or $\mathrm{CH}_{2} \mathrm{Cl}_{2}-\mathrm{MeOH}$ solvent system. All reagents were purchased from commercial sources and used without further purification. Analytical samples of new compounds were obtained by recrystallization from the solvents or solvent mixtures given below in parentheses. The $[\alpha]_{D}$ values were determined as an average value of three measurements, measured in $\mathrm{MeOH}$.

Compounds (rac)-1, $(R)-\mathbf{1}, \mathbf{2},(R)-\mathbf{3},(\mathrm{rac})-\mathbf{9}, 17,18,20,22$ are described in the literature, while compounds $(S)-\mathbf{1},($ rac $)-\mathbf{3},(S)-\mathbf{3},(R)-\mathbf{9}$, $(S)-9,(r a c)-10,(R)-10,(S)-10,(r a c)-11,(R)-11,(S)-11,(r a c)-14,(R)-14$, $(S)-14,($ rac $)-15,(R)-15,(S)-15,(r a c)-16,(R)-16,(S)-16,19,21,25,26$, 27 are novel.

\section{Chromatographic Separation and Conditions}

During chiral separations, a polar organic mode was used on a polysaccharide stationary phase $(150 \times 4.6 \mathrm{~mm}$ Lux $5 \mu \mathrm{m}$ amylose- 1$)$. The column was purchased from Phenomenex (Torrance, USA). The mobile phase used in this work was acetonitrile (gradient grade) with $0.1 \%$ diethylamine. Chemicals were purchased from Merck (Darmstadt, Germany). Chromatographic experiments were performed with a Waters Acquity UPLC H-Class system (Milford, USA) equipped with a quaternary solvent delivery pump, autosampler, photodiode array detector and Empower 3 software. The column temperature was $20^{\circ} \mathrm{C}$. This UHPLC system had a flow-through-needle (FTN) sample injector and $500 \mathrm{~nL}$ flow cell. Before analysis, samples were dissolved in pure acetonitrile $(0.5 \mathrm{mg} / \mathrm{mL})$; injection volume was $1 \mu \mathrm{L}$.

\section{Preparation of Amides 9, 14, 17, 18; General Procedure}

Tryptamine $(8 ; 14 \mathrm{mmol}$ ) and triethylamine (21 mmol, 1.5 equiv) were dissolved in $\mathrm{CH}_{2} \mathrm{Cl}_{2}(80 \mathrm{~mL})$ and cooled in an ice-water bath. To this solution, the appropriate acyl chloride $(15.4 \mathrm{mmol})$ was added dropwise. After addition of the acyl chloride, the reaction mixture was allowed to warm to r.t. and stirred for 2 hours. After the reaction was complete, the mixture was washed with water $(60 \mathrm{~mL}), 5 \% \mathrm{HCl}$ solution $(60 \mathrm{~mL})$ and again with water $(60 \mathrm{~mL})$. The organic phase was dried over $\mathrm{MgSO}_{4}$, filtered and evaporated to provide the amides (9, $\mathbf{1 4}, \mathbf{1 7}, \mathbf{1 8})$. The crude products were purified by flash column chromatography $\left(\mathrm{CH}_{2} \mathrm{Cl}_{2}-\mathrm{MeOH}\right)$.

\section{2-\{[2-(1H-Indol-3-yl)ethyl]amino\}-1-methyl-2-oxoethyl acetate} $[(\text { rac })-9]^{24}$

Yield: $3.047 \mathrm{~g}$ (88\%); brown oil.

IR (KBr): 3405, 3311, 1738, 1667, 1537, 1232, 1096, $744 \mathrm{~cm}^{-1}$.

${ }^{1} \mathrm{H} \mathrm{NMR}\left(600 \mathrm{MHz}, \mathrm{CDCl}_{3}\right): \delta=8.20(\mathrm{br} \mathrm{s}, 1 \mathrm{H}), 7.61(\mathrm{~d}, J=8.0 \mathrm{~Hz}, 1 \mathrm{H})$, $7.39(\mathrm{~d}, J=8.1 \mathrm{~Hz}, 1 \mathrm{H}), 7.26(\mathrm{br} \mathrm{s}, 1 \mathrm{H}), 7.21(\mathrm{t}, J=7.3 \mathrm{~Hz}, 1 \mathrm{H}), 7.14(\mathrm{t}$, $J=7.7 \mathrm{~Hz}, 1 \mathrm{H}), 6.19$ (br s, $1 \mathrm{H}), 5.15(\mathrm{q}, J=6.9 \mathrm{~Hz}, 1 \mathrm{H}), 3.69-3.62(\mathrm{~m}$, $1 \mathrm{H}), 3.61-3.54(\mathrm{~m}, 1 \mathrm{H}), 3.00(\mathrm{t}, J=6.7 \mathrm{~Hz}, 2 \mathrm{H}), 1.93(\mathrm{~s}, 3 \mathrm{H}), 1.43(\mathrm{~d}$, $J=6.8 \mathrm{~Hz}, 3 \mathrm{H})$.

${ }^{13} \mathrm{C}$ NMR $\left(150 \mathrm{MHz}, \mathrm{CDCl}_{3}\right): \delta=170.3,169.4,136.3,127.3,122.23$, 122.16, 119.6, 118.6, 112.7, 111.3, 70.6, 39.5, 25.0, 20.9, 17.9 ppm.

(1R)-2-\{[2-(1H-Indol-3-yl)ethyl]amino\}-1-methyl-2-oxoethyl acetate $[(R)-9]$

Yield: $3.151 \mathrm{~g}$ (91\%); brown oil; $[\alpha]_{\mathrm{D}}^{28}+13.7$ ( c 1.0, MeOH).

IR (film): 3405, 3308, 1735, 1664, 1540, 1233, 1096, $744 \mathrm{~cm}^{-1}$.

${ }^{1} \mathrm{H} \mathrm{NMR}\left(600 \mathrm{MHz}, \mathrm{CDCl}_{3}\right): \delta=8.34(\mathrm{br} \mathrm{s}, 1 \mathrm{H}), 7.60(\mathrm{~d}, J=7.9 \mathrm{~Hz}, 1 \mathrm{H})$, $7.39(\mathrm{~d}, J=8.1 \mathrm{~Hz}, 1 \mathrm{H}), 7.26(\mathrm{br} \mathrm{s}, 1 \mathrm{H}), 7.20(\mathrm{t}, J=7.3 \mathrm{~Hz}, 1 \mathrm{H}), 7.13(\mathrm{t}$, $J=7.8 \mathrm{~Hz}, 1 \mathrm{H}), 7.03(\mathrm{~d}, J=2.0 \mathrm{~Hz}, 1 \mathrm{H}), 6.21(\mathrm{br} \mathrm{s}, 1 \mathrm{H}), 5.15(\mathrm{q}, J=$ $6.8 \mathrm{~Hz}, 1 \mathrm{H}), 3.69-3.62(\mathrm{~m}, 1 \mathrm{H}), 3.61-3.54(\mathrm{~m}, 1 \mathrm{H}), 3.00(\mathrm{t}, J=6.7 \mathrm{~Hz}$, $2 \mathrm{H}), 1.92(\mathrm{~s}, 3 \mathrm{H}), 1.42(\mathrm{~d}, J=6.8 \mathrm{~Hz}, 3 \mathrm{H})$.

${ }^{13} \mathrm{C}$ NMR $\left(150 \mathrm{MHz}, \mathrm{CDCl}_{3}\right): \delta=170.3,169.4,136.2,127.3,122.20$, $122.15,119.5,118.5,112.6,111.3,70.5,39.6,25.0,20.8,17.9$.

HRMS: $m / z[\mathrm{M}+\mathrm{H}]^{+}$calcd for $\mathrm{C}_{15} \mathrm{H}_{19} \mathrm{~N}_{2} \mathrm{O}_{3}$ : 275.1396; found: 275.1390 .

(1S)-2-\{[2-(1H-Indol-3-yl)ethyl]amino\}-1-methyl-2-oxoethyl acetate $[(S)-9]$

Yield: $3.428 \mathrm{~g}$ (99\%); brown oil; $[\alpha]_{\mathrm{D}}^{28}-17.5$ (c 1.0, MeOH).

IR (KBr): 3405, 3306, 1737, 1665, 1537, 1233, 1096, $745 \mathrm{~cm}^{-1}$.

${ }^{1} \mathrm{H} \mathrm{NMR}\left(600 \mathrm{MHz}, \mathrm{CDCl}_{3}\right): \delta=8.19(\mathrm{br} \mathrm{s}, 1 \mathrm{H}), 7.61(\mathrm{~d}, J=7.9 \mathrm{~Hz}, 1 \mathrm{H})$, $7.39(\mathrm{~d}, J=8.1 \mathrm{~Hz}, 1 \mathrm{H}), 7.26(\mathrm{br} \mathrm{s}, 1 \mathrm{H}), 7.22(\mathrm{t}, J=7.2 \mathrm{~Hz}, 1 \mathrm{H}), 7.14(\mathrm{t}$, $J=7.2 \mathrm{~Hz}, 1 \mathrm{H}), 7.04(\mathrm{~d}, J=2.0 \mathrm{~Hz}, 1 \mathrm{H}), 6.19$ (br s, $1 \mathrm{H}), 5.15$ (q, $J=$ $6.8 \mathrm{~Hz}, 1 \mathrm{H}), 3.69-3.62(\mathrm{~m}, 1 \mathrm{H}), 3.61-3.54(\mathrm{~m}, 1 \mathrm{H}), 3.00(\mathrm{t}, J=6.6 \mathrm{~Hz}$, $2 \mathrm{H}), 1.93(\mathrm{~s}, 3 \mathrm{H}), 1.43(\mathrm{~d}, J=6.9 \mathrm{~Hz}, 3 \mathrm{H})$.

${ }^{13} \mathrm{C}$ NMR $\left(150 \mathrm{MHz}, \mathrm{CDCl}_{3}\right): \delta=170.3,169.4,136.3,127.3,122.23$, 122.16, 119.6, 118.6, 112.7, 111.3, 70.6, 39.5, 25.0, 20.9, 17.9.

HRMS: $m / z[\mathrm{M}+\mathrm{H}]^{+}$calcd for $\mathrm{C}_{15} \mathrm{H}_{19} \mathrm{~N}_{2} \mathrm{O}_{3}$ : 275.1396; found: 275.1390 .

\section{1-\{[2-(1 H-Indol-3-yl)ethyl]carbamoyl\}propyl acetate [(rac)-14]}

Yield: $3.994 \mathrm{~g}$ (99\%); brown oil.

IR (film): 3406, 3299, 1739, 1660, 1537, 1233, 745, $426 \mathrm{~cm}^{-1}$.

${ }^{1} \mathrm{H} \mathrm{NMR}\left(600 \mathrm{MHz}, \mathrm{CDCl}_{3}\right): \delta=8.23(\mathrm{br} \mathrm{s}, 1 \mathrm{H}), 7.61(\mathrm{~d}, J=7.9 \mathrm{~Hz}, 1 \mathrm{H})$, $7.38(\mathrm{~d}, J=8.2 \mathrm{~Hz}, 1 \mathrm{H}), 7.26(\mathrm{br} \mathrm{s}, 1 \mathrm{H}), 7.21(\mathrm{t}, J=7.9 \mathrm{~Hz}, 1 \mathrm{H}), 7.14(\mathrm{t}$, $J=7.8 \mathrm{~Hz}, 1 \mathrm{H}), 7.03(\mathrm{~d}, J=2.2 \mathrm{~Hz}, 1 \mathrm{H}), 6.14(\mathrm{br} \mathrm{s}, 1 \mathrm{H}), 5.09$ (q, $J=$ $4.7 \mathrm{~Hz}, 1 \mathrm{H}), 3.69-3.62(\mathrm{~m}, 1 \mathrm{H}), 3.61-3.53(\mathrm{~m}, 1 \mathrm{H}), 3.00(\mathrm{t}, J=6.7 \mathrm{~Hz}$, $2 \mathrm{H}), 1.93(\mathrm{~s}, 3 \mathrm{H}), 1.91-1.85(\mathrm{~m}, 1 \mathrm{H}), 1.84-1.77(\mathrm{~m}, 1 \mathrm{H}), 0.88(\mathrm{t}, J=$ $7.4 \mathrm{~Hz}, 3 \mathrm{H})$

${ }^{13} \mathrm{C}$ NMR $\left(150 \mathrm{MHz}, \mathrm{CDCl}_{3}\right): \delta=169.61,169.57,136.3,127.3,122.3$, $119.5,118.6,112.7,111.3,74.9,39.5,25.1,25.0,20.7,9.0$.

HRMS: $m / z[\mathrm{M}+\mathrm{H}]^{+}$calcd for $\mathrm{C}_{16} \mathrm{H}_{21} \mathrm{~N}_{2} \mathrm{O}_{3}$ : 289.1552; found: 289.1546 . 
(1R)-1-\{[2-(1H-Indol-3-yl)ethyl]carbamoyl\}propyl acetate [(R)-14] Yield: $3.994 \mathrm{~g}$ (99\%); brown oil; $[\alpha]_{\mathrm{D}}^{28}+17.1$ (c 1.0, MeOH). IR (KBr): 3405, 3306, 1738, 1666, 1536, 1232, 745, $426 \mathrm{~cm}^{-1}$. ${ }^{1} \mathrm{H} \mathrm{NMR}\left(600 \mathrm{MHz}, \mathrm{CDCl}_{3}\right): \delta=8.17($ br s, $1 \mathrm{H}), 7.61(\mathrm{~d}, J=7.9 \mathrm{~Hz}, 1 \mathrm{H})$, $7.38(\mathrm{~d}, J=8.2 \mathrm{~Hz}, 1 \mathrm{H}), 7.26$ (br s, $1 \mathrm{H}), 7.23(\mathrm{t}, J=7.9 \mathrm{~Hz}, 1 \mathrm{H}), 7.18(\mathrm{t}$, $J=7.9 \mathrm{~Hz}, 1 \mathrm{H}), 7.04(\mathrm{~d}, J=2.3 \mathrm{~Hz}, 1 \mathrm{H}), 6.13(\mathrm{br} \mathrm{s}, 1 \mathrm{H}), 5.10(\mathrm{q}, J=$ $4.7 \mathrm{~Hz}, 1 \mathrm{H}), 3.69-3.62(\mathrm{~m}, 1 \mathrm{H}), 3.61-3.53(\mathrm{~m}, 1 \mathrm{H}), 3.00(\mathrm{t}, J=6.7 \mathrm{~Hz}$, $2 \mathrm{H}), 1.93$ (s, $3 \mathrm{H}), 1.91-1.85(\mathrm{~m}, 1 \mathrm{H}), 1.84-1.77(\mathrm{~m}, 1 \mathrm{H}), 0.88(\mathrm{t}, J=$ $7.4 \mathrm{~Hz}, 3 \mathrm{H})$.

${ }^{13} \mathrm{C}$ NMR $\left(150 \mathrm{MHz}, \mathrm{CDCl}_{3}\right): \delta=169.59,169.56,136.3,127.3,122.23$, 122.19, 119.6, 118.6, 112.7, 111.3, 74.9, 39.4, 25.1, 25.0, 20.8, 9.0.

HRMS: $m / z[\mathrm{M}+\mathrm{H}]^{+}$calcd for $\mathrm{C}_{16} \mathrm{H}_{21} \mathrm{~N}_{2} \mathrm{O}_{3}$ : 289.1552; found: 289.1547 .

(1S)-1-\{[2-(1H-Indol-3-yl)ethyl]carbamoyl\}propyl acetate [(S)-14] Yield: $3.994 \mathrm{~g}$ (99\%); brown oil; $[\alpha]_{\mathrm{D}}{ }^{28}-20.9$ (c 1.0, MeOH). IR (KBr): 3405, 3307, 1738, 1664, 1536, 1232, 744, $427 \mathrm{~cm}^{-1}$. ${ }^{1} \mathrm{H} \mathrm{NMR}\left(600 \mathrm{MHz}, \mathrm{CDCl}_{3}\right): \delta=8.17(\mathrm{br} \mathrm{s}, 1 \mathrm{H}), 7.61(\mathrm{~d}, J=7.9 \mathrm{~Hz}, 1 \mathrm{H})$, $7.39(\mathrm{~d}, J=8.1 \mathrm{~Hz}, 1 \mathrm{H}), 7.26(\mathrm{br} \mathrm{s}, 1 \mathrm{H}), 7.22(\mathrm{t}, J=7.3 \mathrm{~Hz}, 1 \mathrm{H}), 7.14(\mathrm{t}$, $J=7.8 \mathrm{~Hz}, 1 \mathrm{H}), 7.04(\mathrm{~d}, J=1.9 \mathrm{~Hz}, 1 \mathrm{H}), 6.13(\mathrm{br} \mathrm{s}, 1 \mathrm{H}), 5.10(\mathrm{q}, J=$ $4.8 \mathrm{~Hz}, 1 \mathrm{H}), 3.69-3.62(\mathrm{~m}, 1 \mathrm{H}), 3.61-3.53(\mathrm{~m}, 1 \mathrm{H}), 2.99(\mathrm{t}, J=6.7 \mathrm{~Hz}$, $2 \mathrm{H}), 1.93(\mathrm{~s}, 3 \mathrm{H}), 1.91-1.85(\mathrm{~m}, 1 \mathrm{H}), 1.84-1.77(\mathrm{~m}, 1 \mathrm{H}), 0.88(\mathrm{t}, J=$ $7.4 \mathrm{~Hz}, 3 \mathrm{H})$.

${ }^{13} \mathrm{C}$ NMR $\left(150 \mathrm{MHz}, \mathrm{CDCl}_{3}\right): \delta=169.60,169.57,136.3,127.3,122.23$, $122.20,119.6,118.6,112.7,111.3,74.9,39.5,25.1,25.0,20.8,9.0$.

HRMS: $m / z[\mathrm{M}+\mathrm{H}]^{+}$calcd for $\mathrm{C}_{16} \mathrm{H}_{21} \mathrm{~N}_{2} \mathrm{O}_{3}$ : 289.1552; found: 289.1547 .

Methyl 3-\{[2-(1H-Indol-3-yl)ethyl]amino\}-3-oxopropanoate (17) ${ }^{25}$ Yield: $2.259 \mathrm{~g}(62 \%)$; brown oil.

IR (film): 3393, 3307, 1738, 1655, 1437, 830, 746, $559 \mathrm{~cm}^{-1}$.

${ }^{1} \mathrm{H}$ NMR $\left(600 \mathrm{MHz}, \mathrm{CDCl}_{3}\right): \delta=8.50($ br s, $1 \mathrm{H}), 7.59-7.56(\mathrm{~m}, 1 \mathrm{H})$, 7.34-7.31 (m, $1 \mathrm{H})$, 7.19-7.14 (m, $1 \mathrm{H}), 7.11-7.07$ (m, $1 \mathrm{H}), 7.02$ (br s, $1 \mathrm{H}), 6.98(\mathrm{~d}, J=2.3 \mathrm{~Hz}, 1 \mathrm{H}), 3.65(\mathrm{~s}, 3 \mathrm{H}), 3.59(\mathrm{q}, J=6.9 \mathrm{~Hz}, 2 \mathrm{H}), 3.21$ (s, $2 \mathrm{H}), 2.89-2.94(\mathrm{~m}, 2 \mathrm{H})$

${ }^{13} \mathrm{C}$ NMR $\left(150 \mathrm{MHz}, \mathrm{CDCl}_{3}\right): \delta=169.5,165.0,136.3,127.1,122.2$, $121.9,119.2,118.5,112.4,111.2,52.3,41.2,39.9,24.9$.

\section{$\mathbf{N}$-[2-(1H-Indol-3-yl)ethyl]-3-methylbut-2-enamide (18) ${ }^{26}$}

Yield: $3.359 \mathrm{~g}$ (99\%); colorless crystals; $\mathrm{mp} 73-75^{\circ} \mathrm{C}(\mathrm{EtOH})$. IR (KBr): 3296, 2885, 1626, 1548, 1457, 1222, 931, $799 \mathrm{~cm}^{-1}$.

${ }^{1} \mathrm{H} \mathrm{NMR}\left(600 \mathrm{MHz}, \mathrm{CDCl}_{3}\right): \delta=8.17(\mathrm{br} \mathrm{s}, 1 \mathrm{H}), 7.61(\mathrm{~d}, J=7.9 \mathrm{~Hz}, 1 \mathrm{H})$, $7.38(\mathrm{~d}, J=8.1 \mathrm{~Hz}, 1 \mathrm{H}), 7.26(\mathrm{~s}, 1 \mathrm{H}), 7.21(\mathrm{t}, J=7.4 \mathrm{~Hz}, 1 \mathrm{H}), 7.13(\mathrm{t}, J=$ $7.5 \mathrm{~Hz}, 1 \mathrm{H}), 7.02$ (s, $1 \mathrm{H}), 5.82$ (br s, $1 \mathrm{H}), 4.88(\mathrm{~s}, 1 \mathrm{H}), 4.78(\mathrm{~s}, 1 \mathrm{H})$, $3.60(\mathrm{q}, J=6.4 \mathrm{~Hz}, 2 \mathrm{H}), 2.97(\mathrm{t}, J=6.7 \mathrm{~Hz}, 2 \mathrm{H}), 2.91(\mathrm{~s}, 1 \mathrm{H}), 1.71(\mathrm{~s}$, $3 \mathrm{H})$.

${ }^{13} \mathrm{C}$ NMR $\left(150 \mathrm{MHz}, \mathrm{CDCl}_{3}\right): \delta=170.2,140.3,136.3,127.3,122.2$, 122.0, 119.4, 118.7, 115.5, 112.9, 111.2, 46.3, 39.7, 25.2, 22.4.

\section{Preparation of Amide 25; General Procedure}

Method A: 3-Acetoxy-3-methylbutyric acid (24; $10 \mathrm{mmol})$ and carbonyldiimidazole (CDI; $10 \mathrm{mmol}$ ) were dissolved in $\mathrm{N}$-methyl-2-pyrrolidine $(10 \mathrm{~mL})$ and the mixture was stirred for $1 \mathrm{~h}$. Tryptamine $(\mathbf{8}$; $10 \mathrm{mmol}$ ) was then added in one portion and the reaction mixture was stirred at r.t. overnight. After the conversion was complete, the reaction was quenched with water $(50 \mathrm{~mL})$ and the mixture was extracted with EtOAc $(3 \times 25 \mathrm{~mL})$. The combined organic layers were dried over $\mathrm{MgSO}_{4}$, filtered and evaporated. The crude product was purified with flash column chromatography ( $n$-hexane-EtOAc) for analytical measurements.

Method B: Tryptamine (8; $20 \mathrm{mmol}), 3$-acetoxy-3-methylbutyric acid (24; $20 \mathrm{mmol}$ ), $i$ - $\mathrm{Pr}_{2} \operatorname{EtN}$ ( $40 \mathrm{mmol}, 2$ equiv), and HATU ( $24 \mathrm{mmol}, 1.2$ equiv) were dissolved in THF $(200 \mathrm{~mL})$ and the reaction mixture was stirred at r.t. overnight. After the reaction was complete, the mixture was evaporated. The residue was taken up in $\mathrm{CH}_{2} \mathrm{Cl}_{2}(100 \mathrm{~mL})$ and the organic extract was washed with water $(3 \times 50 \mathrm{~mL})$. The organic phase was dried over $\mathrm{Na}_{2} \mathrm{SO}_{4}$, filtered and evaporated to provide amide $\mathbf{2 5}$. The crude product was purified by flash column chromatography ( $n$-hexane-EtOAc).

3-\{[2-(1H-Indol-3-yl)ethyl]amino\}-1,1-dimethyl-3-oxopropyl acetate (25)

Yield (Method A): $1.149 \mathrm{~g}$ (38\%); Yield (Method B): $3.387 \mathrm{~g}$ (70\%); pale-brown oil.

IR (KBr): 3406, 2935, 1730, 1658, 1369, 1253, 833, $743 \mathrm{~cm}^{-1}$.

${ }^{1} \mathrm{H} \mathrm{NMR}\left(600 \mathrm{MHz}, \mathrm{CDCl}_{3}\right): \delta=8.33(\mathrm{br} \mathrm{s}, 1 \mathrm{H}), 7.60(\mathrm{~d}, J=7.9 \mathrm{~Hz}, 1 \mathrm{H})$, $7.36(\mathrm{~d}, J=8.1 \mathrm{~Hz}, 1 \mathrm{H}), 7.26(\mathrm{~s}, 1 \mathrm{H}), 7.20(\mathrm{t}, J=7.4 \mathrm{~Hz}, 1 \mathrm{H}), 7.12(\mathrm{t}, J=$ $7.6 \mathrm{~Hz}, 1 \mathrm{H}), 7.02(\mathrm{~d}, J=1.8 \mathrm{~Hz}, 1 \mathrm{H}), 5.80(\mathrm{br} \mathrm{s}, 1 \mathrm{H}), 3.61(\mathrm{q}, J=6.5 \mathrm{~Hz}$, $2 \mathrm{H}), 2.96(\mathrm{t}, J=6.8 \mathrm{~Hz}, 2 \mathrm{H}), 2.64(\mathrm{~s}, 2 \mathrm{H}), 1.73(\mathrm{~s}, 3 \mathrm{H}), 1.51(\mathrm{~s}, 6 \mathrm{H})$.

${ }^{13} \mathrm{C}$ NMR $\left(150 \mathrm{MHz}, \mathrm{CDCl}_{3}\right): \delta=170.9,169.4,136.4,127.1,122.2$, 122.0, 119.4, 118.6, 112.6, 111.2, 80.6, 47.2, 39.4, 26.5, 25.3, 22.1.

HRMS: $m / z[\mathrm{M}+\mathrm{H}]^{+}$calcd for $\mathrm{C}_{17} \mathrm{H}_{22} \mathrm{~N}_{2} \mathrm{O}_{3}$ : 303.1709; found: 303.1703 .

\section{Preparation of Ketoamides 10, 15, 19, 20, 26; General Procedure}

The appropriate amide $\mathbf{9}, \mathbf{1 4}, \mathbf{1 7}, \mathbf{1 8}, 25$ (4 mmol) was dissolved in THF- $\mathrm{H}_{2} \mathrm{O}(9: 1,60 \mathrm{~mL})$. To this solution was added 2,3-dichloro-5,6dicyano-1,4-benzoquinone (DDQ; $8 \mathrm{mmol}, 2$ equiv) in several portions at $0{ }^{\circ} \mathrm{C}$. After addition was complete, the mixture was stirred at the same temperature for $2 \mathrm{~h}$ (until the consumption of the starting material as indicated by TLC). The mixture was then poured into EtOAc $(150 \mathrm{~mL})$, extracted with $10 \%$ aq. $\mathrm{NaHCO}_{3}(4 \times 85 \mathrm{~mL})$, dried over $\mathrm{MgSO}_{4}$, filtered, and evaporated to give the desired ketoamide. Analytical samples were obtained by recrystallization from EtOH.

2-\{[2-(1H-Indol-3-yl)-2-oxoethyl]amino\}-1-methyl-2-oxoethyl acetate $[($ rac $)-10]$

Yield: $1.096 \mathrm{~g}$ (95\%); colorless crystals; $\mathrm{mp} 128-130{ }^{\circ} \mathrm{C}(\mathrm{EtOH})$. IR (KBr): 3278, 3116, 1737, 1666, 1633, 1243, 1048, $756 \mathrm{~cm}^{-1}$.

${ }^{1} \mathrm{H}$ NMR $\left(600 \mathrm{MHz}\right.$, DMSO- $\left.d_{6}\right): \delta=12.03(\mathrm{br} \mathrm{s}, 1 \mathrm{H}), 8.43(\mathrm{~d}, J=3.1 \mathrm{~Hz}$, $1 \mathrm{H}), 8.30(\mathrm{t}, J=5.6 \mathrm{~Hz}, 1 \mathrm{H}), 8.16(\mathrm{~d}, J=7.4 \mathrm{~Hz}, 1 \mathrm{H}), 7.49(\mathrm{~d}, J=7.9 \mathrm{~Hz}$, $1 \mathrm{H}), 7.36-7.27(\mathrm{~m}, 2 \mathrm{H}), 5.07(\mathrm{q}, J=6.9 \mathrm{~Hz}, 1 \mathrm{H}), 4.48(\mathrm{~d}, J=6.9 \mathrm{~Hz}$, $2 \mathrm{H}), 2.10(\mathrm{~s}, 3 \mathrm{H}), 1.39(\mathrm{~d}, J=7.2 \mathrm{~Hz}, 3 \mathrm{H})$.

${ }^{13} \mathrm{C}$ NMR $\left(150 \mathrm{MHz}\right.$, DMSO- $\left.d_{6}\right): \delta=190.0,170.5,169.9,136.6,133.9$, 125.6, 123.1, 122.1, 121.3, 114.1, 112.4, 69.8, 45.6, 21.0, 18.1.

HRMS: $m / z[\mathrm{M}+\mathrm{H}]^{+}$calcd for $\mathrm{C}_{15} \mathrm{H}_{17} \mathrm{~N}_{2} \mathrm{O}_{4}$ : 289.1188; found: 289.1183 .

(1R)-2-\{[2-(1H-Indol-3-yl)-2-oxoethyl]amino\}-1-methyl-2-oxoethyl acetate $[(R)-10]$

Yield: $1.049 \mathrm{~g}$ (91\%); colorless crystals; mp $128-130{ }^{\circ} \mathrm{C}(\mathrm{EtOH}) ;[\alpha]_{\mathrm{D}}{ }^{28}$ +31.6 (c 1.0, MeOH).

IR (KBr): 3386, 3258, 1743, 1662, 1631, 1528, 1230, $745 \mathrm{~cm}^{-1}$.

${ }^{1} \mathrm{H}$ NMR $\left(600 \mathrm{MHz}\right.$, DMSO- $\left.d_{6}\right): \delta=12.03(\mathrm{br} \mathrm{s}, 1 \mathrm{H}), 8.44(\mathrm{~d}, J=3.1 \mathrm{~Hz}$, $1 \mathrm{H}), 8.31(\mathrm{t}, J=5.6 \mathrm{~Hz}, 1 \mathrm{H}), 8.17(\mathrm{~d}, J=7.5 \mathrm{~Hz}, 1 \mathrm{H}), 7.49(\mathrm{~d}, J=7.7 \mathrm{~Hz}$, $1 \mathrm{H}), 7.26-7.17(\mathrm{~m}, 2 \mathrm{H}), 5.07(\mathrm{q}, J=6.8 \mathrm{~Hz}, 1 \mathrm{H}), 4.48(\mathrm{~d}, J=5.8 \mathrm{~Hz}$, $2 \mathrm{H}), 2.10(\mathrm{~s}, 3 \mathrm{H}), 1.40(\mathrm{~d}, J=6.8 \mathrm{~Hz}, 3 \mathrm{H})$. 
${ }^{13} \mathrm{C}$ NMR $\left(150 \mathrm{MHz}\right.$, DMSO- $\left.d_{6}\right): \delta=190.0,170.6,169.9,136.6,133.9$, 125.6, 123.1, 122.1, 121.3, 114.1, 112.4, 69.8, 45.6, 21.0, 18.1.

HRMS: $m / z[\mathrm{M}+\mathrm{H}]^{+}$calcd for $\mathrm{C}_{15} \mathrm{H}_{17} \mathrm{~N}_{2} \mathrm{O}_{4}$ : 289.1188; found: 289.1183 .

(1S)-2-\{[2-(1H-Indol-3-yl)-2-oxoethyl]amino\}-1-methyl-2-oxoethyl acetate $[(S)-10]$

Yield: $1.072 \mathrm{~g}$ (93\%); colorless crystals; mp $134-136{ }^{\circ} \mathrm{C}\left(\right.$ EtOH); $[\alpha]_{D}{ }^{28}$ -30.9 (c 1.0, MeOH).

IR (KBr): 3332, 3260, 1744, 1659, 1621, 1228, 1120, $746 \mathrm{~cm}^{-1}$.

${ }^{1} \mathrm{H}$ NMR $\left(600 \mathrm{MHz}\right.$, DMSO- $\left.d_{6}\right): \delta=12.03(\mathrm{br} \mathrm{s}, 1 \mathrm{H}), 8.44(\mathrm{~s}, 1 \mathrm{H}), 8.30$ $(\mathrm{t}, J=5.7 \mathrm{~Hz}, 1 \mathrm{H}), 8.16(\mathrm{~d}, J=7.2 \mathrm{~Hz}, 1 \mathrm{H}), 7.48(\mathrm{~d}, J=7.8 \mathrm{~Hz}, 1 \mathrm{H})$, 7.26-7.17 (m, $2 \mathrm{H}), 5.07(\mathrm{q}, J=6.8 \mathrm{~Hz}, 1 \mathrm{H}), 4.48(\mathrm{~d}, J=5.7 \mathrm{~Hz}, 2 \mathrm{H})$, $2.09(\mathrm{~s}, 3 \mathrm{H}), 1.39(\mathrm{~d}, J=6.8 \mathrm{~Hz}, 3 \mathrm{H})$.

${ }^{13} \mathrm{C}$ NMR $\left(150 \mathrm{MHz}\right.$, DMSO- $\left.d_{6}\right): \delta=190.0,170.5,169.9,136.6,133.9$, 125.6, 123.1, 122.1, 121.3, 114.1, 112.4, 69.8, 45.6, 21.0, 18.1.

HRMS: $m / z[\mathrm{M}+\mathrm{H}]^{+}$calcd for $\mathrm{C}_{15} \mathrm{H}_{17} \mathrm{~N}_{2} \mathrm{O}_{4}$ : 289.1188; found: 289.1183 .

1-\{[2-(1H-Indol-3-yl)-2-oxoethyl]carbamoyl\}propyl acetate [(rac)15]

Yield: $1.197 \mathrm{~g}$ (99\%); colorless crystals; mp $152-153^{\circ} \mathrm{C}(\mathrm{EtOH})$.

IR (KBr): 3275, 3106, 1733, 1664, 1631, 1580, 1236, $757 \mathrm{~cm}^{-1}$.

${ }^{1} \mathrm{H}$ NMR $\left(600 \mathrm{MHz}\right.$, DMSO- $\left.d_{6}\right): \delta=12.02(\mathrm{br} \mathrm{s}, 1 \mathrm{H}), 8.44(\mathrm{~d}, J=3.1 \mathrm{~Hz}$, $1 \mathrm{H}), 8.29(\mathrm{t}, J=5.7 \mathrm{~Hz}, 1 \mathrm{H}), 8.16(\mathrm{~d}, J=7.6 \mathrm{~Hz}, 1 \mathrm{H}), 7.48(\mathrm{~d}, J=7.9 \mathrm{~Hz}$, $1 \mathrm{H}), 7.26-7.17(\mathrm{~m}, 2 \mathrm{H}), 4.96(\mathrm{q}, J=4.9 \mathrm{~Hz}, 1 \mathrm{H}), 4.55-4.46(\mathrm{~m}, 2 \mathrm{H})$, $2.11(\mathrm{~s}, 3 \mathrm{H}), 1.88-1.71(\mathrm{~m}, 2 \mathrm{H}), 1.39(\mathrm{t}, J=7.4 \mathrm{~Hz}, 3 \mathrm{H})$.

${ }^{13} \mathrm{C}$ NMR $\left(150 \mathrm{MHz}\right.$, DMSO- $\left.d_{6}\right): \delta=190.0,170.1,169.7,136.6,133.9$, $125.6,123.1,122.1,121.3,114.2,112.4,74.4,45.6,25.1,21.0,9.5$.

HRMS: $m / z[\mathrm{M}+\mathrm{H}]^{+}$calcd for $\mathrm{C}_{16} \mathrm{H}_{19} \mathrm{~N}_{2} \mathrm{O}_{4}$ : 303.1345; found: 303.1339 .

\section{(1R)-1-\{[2-(1H-Indol-3-yl)-2-oxoethyl]carbamoyl $\}$ propyl acetate [(R)-15]}

Yield: $1.247 \mathrm{~g}$ (93\%); colorless crystals; $\mathrm{mp} 154-157{ }^{\circ} \mathrm{C}(\mathrm{EtOH}) ;[\alpha]_{\mathrm{D}}{ }^{28}$ +36.5 (c 1.0, MeOH)

IR (KBr): 3346, 3277, 1746, 1628, 1520, 1431, 1232, $746 \mathrm{~cm}^{-1}$.

${ }^{1} \mathrm{H}$ NMR $\left(600 \mathrm{MHz}\right.$, DMSO- $\left.d_{6}\right): \delta=12.02(\mathrm{br} \mathrm{s}, 1 \mathrm{H}), 8.44(\mathrm{~d}, J=3.2 \mathrm{~Hz}$, $1 \mathrm{H}), 8.29(\mathrm{t}, J=5.7 \mathrm{~Hz}, 1 \mathrm{H}), 8.16(\mathrm{~d}, J=7.2 \mathrm{~Hz}, 1 \mathrm{H}), 7.48(\mathrm{~d}, J=7.8 \mathrm{~Hz}$, $1 \mathrm{H}), 7.26-7.17(\mathrm{~m}, 2 \mathrm{H}), 4.96(\mathrm{q}, J=4.9 \mathrm{~Hz}, 1 \mathrm{H}), 4.52-4.48(\mathrm{~m}, 2 \mathrm{H})$, $2.11(\mathrm{~s}, 3 \mathrm{H}), 1.88-1.71(\mathrm{~m}, 2 \mathrm{H}), 0.95(\mathrm{t}, J=7.6 \mathrm{~Hz}, 3 \mathrm{H})$.

${ }^{13} \mathrm{C}$ NMR (150 MHz, DMSO- $\left.d_{6}\right): \delta=190.0,170.1,169.7,136.6,133.9$, 125.6, 123.1, 122.1, 121.3, 114.2, 112.4, 74.4, 45.6, 25.1, 21.0, 9.5.

HRMS: $m / z[\mathrm{M}+\mathrm{H}]^{+}$calcd for $\mathrm{C}_{16} \mathrm{H}_{19} \mathrm{~N}_{2} \mathrm{O}_{4}$ : 303.1345; found: 303.1339 .

\section{(1S)-1-\{[2-(1H-Indol-3-yl)-2-oxoethyl]carbamoyl\}propyl acetate [(S)-15]}

Yield: $1.197 \mathrm{~g}(99 \%)$; colorless crystals; mp $151-154{ }^{\circ} \mathrm{C}(\mathrm{EtOH}) ;[\alpha]_{\mathrm{D}}{ }^{28}$ -34.8 (c 1.0, $\mathrm{MeOH}$ ).

IR (KBr): 3346, 3276, 1746, 1627, 1519, 1430, 1232, $746 \mathrm{~cm}^{-1}$

${ }^{1} \mathrm{H}$ NMR $\left(600 \mathrm{MHz}\right.$, DMSO- $\left.d_{6}\right): \delta=12.02$ (br s, $\left.1 \mathrm{H}\right), 8.44(\mathrm{~d}, J=3.2 \mathrm{~Hz}$, $1 \mathrm{H}), 8.29(\mathrm{t}, J=5.7 \mathrm{~Hz}, 1 \mathrm{H}), 8.16(\mathrm{~d}, J=7.2 \mathrm{~Hz}, 1 \mathrm{H}), 7.48(\mathrm{~d}, J=7.8 \mathrm{~Hz}$, $1 \mathrm{H}), 7.26-7.17(\mathrm{~m}, 2 \mathrm{H}), 4.96(\mathrm{q}, J=4.9 \mathrm{~Hz}, 1 \mathrm{H}), 4.52-4.48(\mathrm{~m}, 2 \mathrm{H})$, $2.11(\mathrm{~s}, 3 \mathrm{H}), 1.88-1.71(\mathrm{~m}, 2 \mathrm{H}), 0.95(\mathrm{t}, J=7.4 \mathrm{~Hz}, 3 \mathrm{H})$.

${ }^{13} \mathrm{C}$ NMR $\left(150 \mathrm{MHz}\right.$, DMSO- $\left.d_{6}\right): \delta=190.0,170.1,169.7,136.6,133.9$, 125.6, 123.1, 122.1, 121.3, 114.2, 112.4, 74.4, 45.6, 25.1, 21.0, 9.5

HRMS: $m / z[\mathrm{M}+\mathrm{H}]^{+}$calcd for $\mathrm{C}_{16} \mathrm{H}_{19} \mathrm{~N}_{2} \mathrm{O}_{4}$ : 303.1345; found: 303.1339 .
Methyl 3-\{[2-(1H-Indol-3-yl)-2-oxoethyl]amino\}-3-oxopropanoate (19)

Yield: $1.086 \mathrm{~g}$ (99\%); pale-brown crystals; mp $173-176{ }^{\circ} \mathrm{C}(\mathrm{EtOH})$. IR (KBr): 3302, 3219, 1741, 1667, 1625, 1437, 1208, $743 \mathrm{~cm}^{-1}$. ${ }^{1} \mathrm{H} \mathrm{NMR}\left(600 \mathrm{MHz}, \mathrm{CDCl}_{3}\right): \delta=8.86(\mathrm{br} \mathrm{s}, 1 \mathrm{H}), 8.35(\mathrm{t}, J=3.7 \mathrm{~Hz}, 1 \mathrm{H})$, $7.96(\mathrm{~d}, J=3.1 \mathrm{~Hz}, 2 \mathrm{H}), 7.49-7.43(\mathrm{~m}, 1 \mathrm{H}), 7.37-7.30(\mathrm{~m}, 2 \mathrm{H}), 4.70$ $(\mathrm{d}, J=4.3 \mathrm{~Hz}, 2 \mathrm{H}), 3.80(\mathrm{~s}, 3 \mathrm{H}), 3.45(\mathrm{~s}, 2 \mathrm{H}), 3.59(\mathrm{q}, J=6.9 \mathrm{~Hz}, 2 \mathrm{H})$, 3.21 (s, $2 \mathrm{H}), 2.89-2.94(\mathrm{~m}, 2 \mathrm{H})$.

${ }^{13} \mathrm{C}$ NMR $\left(150 \mathrm{MHz}, \mathrm{CDCl}_{3}\right): \delta=188.4,169.2,165.1,136.1,131.1$, 125.2, 124.1, 123.1, 122.2, 115.4, 111.6, 52.6, 46.6, 41.3.

HRMS: $m / z$ [M+ H] $]^{+}$calcd for $\mathrm{C}_{14} \mathrm{H}_{14} \mathrm{~N}_{2} \mathrm{O}_{4}$ : 275.0954; found: 275.1024 .

$\mathrm{N}$-[2-(1H-Indol-3-yl)-2-oxoethyl]-3-methylbut-2-enamide (20)

Yield: $1.015 \mathrm{~g}$ (99\%); colorless crystals; mp $243-245{ }^{\circ} \mathrm{C}(\mathrm{EtOH}$, decomp.) (lit. ${ }^{7} \mathrm{mp} 230-233^{\circ} \mathrm{C}$ ).

IR (KBr): 3335, 3221, 1624, 1536, 1515, 1435, 926, $742 \mathrm{~cm}^{-1}$.

${ }^{1} \mathrm{H} \mathrm{NMR}\left(400 \mathrm{MHz}\right.$, DMSO- $\left.d_{6}\right): \delta=11.99$ (br s, $\left.1 \mathrm{H}\right), 8.43(\mathrm{~s}, 1 \mathrm{H}), 8.22-$ $8.12(\mathrm{~m}, 1 \mathrm{H}), 8.05(\mathrm{t}, J=5.6 \mathrm{~Hz}, 1 \mathrm{H}), 7.53-7.45(\mathrm{~m}, 1 \mathrm{H}), 7.28-7.16$ (m, $2 \mathrm{H}), 5.81(\mathrm{~s}, 1 \mathrm{H}), 4.48(\mathrm{~d}, J=5.8 \mathrm{~Hz}, 1 \mathrm{H}), 2.10(\mathrm{~s}, 3 \mathrm{H}), 1.81(\mathrm{~s}$, $3 \mathrm{H})$.

${ }^{13} \mathrm{C}$ NMR $\left(100 \mathrm{MHz}\right.$, DMSO- $\left.d_{6}\right): \delta=190.8,166.4,149.0,136.6,133.7$, 125.6, 123.0, 122.0, 121.3, 119.1, 114.3, 112.3, 45.6, 27.0, 19.5.

3-\{[2-(1H-Indol-3-yl)ethyl]amino\}-1,1-dimethyl-3-oxopropyl acetate (26)

Yield: 696 mg (55\%); colorless crystals; mp $169-171{ }^{\circ} \mathrm{C}($ EtOH, decomp.).

IR (KBr): 3328, 3243, 1737, 1630, 1431, 1251, 1144, $746 \mathrm{~cm}^{-1}$.

${ }^{1} \mathrm{H}$ NMR $\left(600 \mathrm{MHz}\right.$, DMSO- $\left.d_{6}\right): \delta=12.01$ (br s, $1 \mathrm{H}$ ), 8.43 (s, $1 \mathrm{H}$ ), 8.20$8.12(\mathrm{~m}, 2 \mathrm{H}), 7.52-7.47(\mathrm{~m}, 1 \mathrm{H}), 7.25-7.17(\mathrm{~m}, 2 \mathrm{H}), 4.47(\mathrm{~d}, J=$ $5.6 \mathrm{~Hz}, 1 \mathrm{H}), 2.74(\mathrm{~s}, 2 \mathrm{H}), 1.93(\mathrm{~s}, 3 \mathrm{H}), 1.51(\mathrm{~s}, 6 \mathrm{H})$.

${ }^{13} \mathrm{C}$ NMR $\left(150 \mathrm{MHz}\right.$, DMSO- $\left.d_{6}\right): \delta=190.4,170.1,169.1,136.6,133.8$, 125.6, 123.1, 122.0, 121.4, 114.2, 112.4, 80.2, 45.9, 45.2, 26.5, 22.5.

HRMS: $m / z[\mathrm{M}+\mathrm{H}]^{+}$calcd for $\mathrm{C}_{17} \mathrm{H}_{21} \mathrm{~N}_{2} \mathrm{O}_{4}$ : 317.1501; found: 317.1496 .

Formation of Oxazoles 11, 16, 27 in the Presence of $\mathrm{POCl}_{3}$ and Pyridine; General Procedure

Ketoamide (10, 15, 26; $0.6 \mathrm{mmol})$ was dissolved in pyridine $(2 \mathrm{~mL})$, $\mathrm{POCl}_{3}$ (3.24 mmol, 5.4 equiv) was added at r.t. and the reaction mixture was stirred 3 hours. After the reaction was complete (TLC monitoring), EtOAc $(40 \mathrm{~mL})$ and $10 \%$ aq. $\mathrm{NaHCO}_{3}(60 \mathrm{~mL})$ were added to the mixture, the phases were separated, and the aqueous phase was further extracted with EtOAc $(3 \times 40 \mathrm{~mL})$. The combined organic extracts were washed with water $(60 \mathrm{~mL})$ and brine $(60 \mathrm{~mL})$, then dried $\left(\mathrm{MgSO}_{4}\right)$, filtered and evaporated to afford the appropriate products. Analytical samples were obtained by recrystallization from aqueous EtOH.

\section{1-[5-(1H-Indol-3-yl)-1,3-oxazol-2-yl]ethyl acetate [(rac)-11]}

Yield: $130 \mathrm{mg}(80 \%)$; pale-brown crystals; mp $139-141{ }^{\circ} \mathrm{C}$ (EtOH$\mathrm{H}_{2} \mathrm{O}$ ).

IR (KBr): 3473, 3171, 1750, 1634, 1495, 1221, 1044, $741 \mathrm{~cm}^{-1}$. ${ }^{1} \mathrm{H}$ NMR $\left(600 \mathrm{MHz}, \mathrm{CD}_{3} \mathrm{OD}\right): \delta=7.80\left(\mathrm{dd},{ }^{1} J=0.4 \mathrm{~Hz},{ }^{2} J=7.9 \mathrm{~Hz}, 1 \mathrm{H}\right)$, $7.65(\mathrm{~s}, 1 \mathrm{H}), 7.44(\mathrm{~d}, J=8.1 \mathrm{~Hz}, 1 \mathrm{H}), 7.25(\mathrm{~s}, 1 \mathrm{H}), 7.20$ (t, $J=7.1 \mathrm{~Hz}$, $1 \mathrm{H}), 7.16(\mathrm{t}, J=7.8 \mathrm{~Hz}, 1 \mathrm{H}), 6.01(\mathrm{q}, J=6.8 \mathrm{~Hz}, 1 \mathrm{H}), 2.12(\mathrm{~s}, 3 \mathrm{H}), 1.69$ (d, $J=6.7 \mathrm{~Hz}, 3 \mathrm{H})$. 
${ }^{13} \mathrm{C}$ NMR (150 MHz, $\left.\mathrm{CD}_{3} \mathrm{OD}\right): \delta=171.6,161.3,151.0,138.2,125.4$, $124.3,123.5,121.5,120.4,119.5,112.9,105.0,66.2,20.8,18.6 \mathrm{ppm}$. HRMS: $m / z[\mathrm{M}+\mathrm{H}]^{+}$calcd for $\mathrm{C}_{15} \mathrm{H}_{15} \mathrm{~N}_{2} \mathrm{O}_{3}$ : 271.1083; found: 271.1077 .

(1R)-1-[5-(1H-Indol-3-yl)-1,3-oxazol-2-yl]ethyl acetate [(R)-11] Yield: $125 \mathrm{mg}$ (77\%); pale-brown crystals; $\mathrm{mp} 164-166{ }^{\circ} \mathrm{C}$ (EtOH$\mathrm{H}_{2} \mathrm{O}$ ); $[\alpha]_{\mathrm{D}}{ }^{28}+87.2$ (c $\left.1.0, \mathrm{MeOH}\right)$.

IR (KBr): 3472, 3169, 1753, 1637, 1494, 1222, 1066, $749 \mathrm{~cm}^{-1}$. ${ }^{1} \mathrm{H}$ NMR (600 MHz, CD $\left.\mathrm{OD}\right): \delta=7.81(\mathrm{~d} J=7.9 \mathrm{~Hz}, 1 \mathrm{H}), 7.65(\mathrm{~s}, 1 \mathrm{H})$, $7.44(\mathrm{~d}, J=8.1 \mathrm{~Hz}, 1 \mathrm{H}), 7.25(\mathrm{~s}, 1 \mathrm{H}), 7.24-7.19(\mathrm{~m}, 1 \mathrm{H}), 7.18-7.14(\mathrm{~m}$, $1 \mathrm{H}), 6.02(\mathrm{q}, J=6.8 \mathrm{~Hz}, 1 \mathrm{H}), 2.13(\mathrm{~s}, 3 \mathrm{H}), 1.69(\mathrm{~d}, J=6.7 \mathrm{~Hz}, 3 \mathrm{H})$.

${ }^{13} \mathrm{C}$ NMR $\left(150 \mathrm{MHz}, \mathrm{CD}_{3} \mathrm{OD}\right): \delta=171.6,161.3,151.0,138.2,125.3$, $124.3,123.5,121.5,120.4,119.5,112.9,105.0,66.2,20.8,18.6$.

HRMS: $m / z[\mathrm{M}+\mathrm{H}]^{+}$calcd for $\mathrm{C}_{15} \mathrm{H}_{15} \mathrm{~N}_{2} \mathrm{O}_{3}$ : 271.1083; found: 271.1077 .

\section{(1S)-1-[5-(1H-Indol-3-yl)-1,3-oxazol-2-yl]ethyl acetate [(S)-11]}

Yield: $120 \mathrm{mg}$ (74\%); pale-brown crystals; mp $164-167{ }^{\circ} \mathrm{C}$ (EtOH$\mathrm{H}_{2} \mathrm{O}$ ); $[\alpha]_{\mathrm{D}}^{28}-89.4$ (c $\left.1.0, \mathrm{MeOH}\right)$.

IR (KBr): 3473, 3169, 1753, 1637, 1451, 1222, 1066, $749 \mathrm{~cm}^{-1}$.

${ }^{1} \mathrm{H}$ NMR $\left(600 \mathrm{MHz}, \mathrm{CD}_{3} \mathrm{OD}\right): \delta=7.80(\mathrm{~d} J=7.9 \mathrm{~Hz}, 1 \mathrm{H}), 7.65(\mathrm{~s}, 1 \mathrm{H})$, $7.46(\mathrm{~d}, J=8.1 \mathrm{~Hz}, 1 \mathrm{H}), 7.26(\mathrm{~s}, 1 \mathrm{H}), 7.24-7.19(\mathrm{~m}, 1 \mathrm{H}), 7.18-7.14(\mathrm{~m}$, $1 \mathrm{H}), 6.02(\mathrm{q}, J=6.8 \mathrm{~Hz}, 1 \mathrm{H}), 2.13(\mathrm{~s}, 3 \mathrm{H}), 1.69(\mathrm{~d}, J=6.7 \mathrm{~Hz}, 3 \mathrm{H})$.

${ }^{13} \mathrm{C}$ NMR (150 MHz, $\left.\mathrm{CD}_{3} \mathrm{OD}\right): \delta=171.6,161.3,151.0,138.2,125.3$, $124.3,123.5,121.5,120.4,119.5,112.9,105.0,66.2,20.8,18.6$.

HRMS: $m / z[\mathrm{M}+\mathrm{H}]^{+}$calcd for $\mathrm{C}_{15} \mathrm{H}_{15} \mathrm{~N}_{2} \mathrm{O}_{3}$ : 271.1083; found: 271.1077 .

\section{1-[5-(1H-Indol-3-yl)-1,3-oxazol-2-yl]propyl acetate [(rac)-16]} Yield: $162 \mathrm{mg}$ (95\%); pale-yellow crystals; mp 131-133 ${ }^{\circ} \mathrm{C}$ (EtOH$\left.\mathrm{H}_{2} \mathrm{O}\right)$.

IR (KBr): 3454, 3184, 1750, 1636, 1453, 1230, 1119, $739 \mathrm{~cm}^{-1}$.

${ }^{1} \mathrm{H}$ NMR $\left(600 \mathrm{MHz}, \mathrm{CD}_{3} \mathrm{OD}\right): \delta=7.79(\mathrm{~d} J=7.9 \mathrm{~Hz}, 1 \mathrm{H}), 7.65(\mathrm{~s}, 1 \mathrm{H})$, $7.40(\mathrm{~d}, J=8.1 \mathrm{~Hz}, 1 \mathrm{H}), 7.26(\mathrm{~s}, 1 \mathrm{H}), 7.21(\mathrm{t}, J=7.7 \mathrm{~Hz}, 1 \mathrm{H}), 7.16(\mathrm{t}, J=$ $7.9 \mathrm{~Hz}, 1 \mathrm{H}), 5.82(\mathrm{t}, J=6.9 \mathrm{~Hz}, 1 \mathrm{H}), 2.14(\mathrm{~s}, 3 \mathrm{H}), 2.13-2.03(\mathrm{~m}, 2 \mathrm{H})$, $1.01(\mathrm{t}, J=7.4 \mathrm{~Hz}, 3 \mathrm{H})$.

${ }^{13} \mathrm{C}$ NMR $\left(150 \mathrm{MHz}, \mathrm{CD}_{3} \mathrm{OD}\right): \delta=171.8,160.7,150.9,138.2,125.3$, 124.3, 123.5, 121.5, 120.4, 119.5, 112.9, 105.0, 71.0, 27.1, 20.7, 9.7.

HRMS: $m / z[\mathrm{M}+\mathrm{H}]^{+}$calcd for $\mathrm{C}_{16} \mathrm{H}_{17} \mathrm{~N}_{2} \mathrm{O}_{3}$ : 285.1239; found: 285.1234 .

(1R)-1-[5-(1H-Indol-3-yl)-1,3-oxazol-2-yl]propyl acetate [(R)-16] Yield: $163 \mathrm{mg}$ (96\%); pale-brown crystals; mp $115-117^{\circ} \mathrm{C}$ (EtOH$\mathrm{H}_{2} \mathrm{O}$ ); $[\alpha]_{\mathrm{D}}{ }^{28}+97.3$ (c $\left.0.5, \mathrm{MeOH}\right)$.

IR (KBr): 3379, 3183, 1735, 1631, 1460, 1220, 1016, $743 \mathrm{~cm}^{-1}$.

${ }^{1} \mathrm{H}$ NMR (600 MHz, CD $\left.\mathrm{OD}\right): \delta=7.80(\mathrm{~d} J=7.9 \mathrm{~Hz}, 1 \mathrm{H}), 7.65(\mathrm{~s}, 1 \mathrm{H})$, $7.44(\mathrm{~d}, J=8.1 \mathrm{~Hz}, 1 \mathrm{H}), 7.26(\mathrm{~s}, 1 \mathrm{H}), 7.23-7.19(\mathrm{~m}, 1 \mathrm{H}), 7.18-7.14(\mathrm{~m}$, $1 \mathrm{H}), 5.83(\mathrm{t}, J=6.7 \mathrm{~Hz}, 1 \mathrm{H}), 2.14(\mathrm{~s}, 3 \mathrm{H}), 2.13-2.03(\mathrm{~m}, 2 \mathrm{H}), 1.01(\mathrm{t}$, $J=7.4 \mathrm{~Hz}, 3 \mathrm{H})$.

${ }^{13} \mathrm{C}$ NMR $\left(150 \mathrm{MHz}, \mathrm{CD}_{3} \mathrm{OD}\right): \delta=171.8,160.7,150.9,138.2,125.3$, 124.3, 123.5, 121.5, 120.4, 119.5, 112.9, 105.0, 71.0, 27.1, 20.7, 9.7.

HRMS: $m / z[\mathrm{M}+\mathrm{H}]^{+}$calcd for $\mathrm{C}_{16} \mathrm{H}_{17} \mathrm{~N}_{2} \mathrm{O}_{3}$ : 285.1239; found: 285.1234 .

(1S)-1-[5-(1H-Indol-3-yl)-1,3-oxazol-2-yl]propyl acetate [(S)-16] Yield: $157 \mathrm{mg}$ (92\%); pale-yellow crystals; mp $116-117^{\circ} \mathrm{C}$ (EtOH$\mathrm{H}_{2} \mathrm{O}$ ); $[\alpha]_{\mathrm{D}}{ }^{28}-102.4$ (c 0.5, MeOH).
IR (KBr): 3379, 3183, 1735, 1631, 1460, 1221, 1016, $743 \mathrm{~cm}^{-1}$. ${ }^{1} \mathrm{H}$ NMR $\left(600 \mathrm{MHz}, \mathrm{CD}_{3} \mathrm{OD}\right): \delta=7.80(\mathrm{~d}, J=7.9 \mathrm{~Hz}, 1 \mathrm{H}), 7.65(\mathrm{~s}, 1 \mathrm{H})$, $7.44(\mathrm{~d}, J=8.1 \mathrm{~Hz}, 1 \mathrm{H}), 7.26(\mathrm{~s}, 1 \mathrm{H}), 7.23-7.19(\mathrm{~m}, 1 \mathrm{H}), 7.18-7.14(\mathrm{~m}$, $1 \mathrm{H}), 5.83(\mathrm{t}, J=6.7 \mathrm{~Hz}, 1 \mathrm{H}), 2.14(\mathrm{~s}, 3 \mathrm{H}), 2.13-2.03(\mathrm{~m}, 2 \mathrm{H}), 1.01(\mathrm{t}$, $J=7.4 \mathrm{~Hz}, 3 \mathrm{H})$.

${ }^{13} \mathrm{C}$ NMR (150 MHz, $\left.\mathrm{CD}_{3} \mathrm{OD}\right): \delta=171.8,160.7,150.9,138.2,125.3$, 124.3, 123.5, 121.5, 120.4, 119.5, 112.9, 105.0, 71.0, 27.1, 20.7, 9.7.

HRMS: $m / z[\mathrm{M}+\mathrm{H}]^{+}$calcd for $\mathrm{C}_{16} \mathrm{H}_{17} \mathrm{~N}_{2} \mathrm{O}_{3}$ : 285.1239; found: 285.1234 .

\section{2-[5-(1H-Indol-3-yl)-1,3-oxazol-2-yl]-1,1-dimethylethyl acetate (27)}

Yield: $170 \mathrm{mg}$ (95\%); colorless crystals; $\mathrm{mp} 145-147{ }^{\circ} \mathrm{C}\left(\mathrm{EtOH}-\mathrm{H}_{2} \mathrm{O}\right)$. IR (KBr): 3445, 3209, 1731, 1604, 1428, 1255, 1172, $735 \mathrm{~cm}^{-1}$.

${ }^{1} \mathrm{H}$ NMR $\left(600 \mathrm{MHz}, \mathrm{CD}_{3} \mathrm{OD}\right): \delta=7.82(\mathrm{~d}, J=7.9 \mathrm{~Hz}, 1 \mathrm{H}), 7.60(\mathrm{~s}, 1 \mathrm{H})$, $7.44(\mathrm{~d}, J=8.1 \mathrm{~Hz}, 1 \mathrm{H}), 7.24-7.18(\mathrm{~m}, 2 \mathrm{H}), 7.17-7.13(\mathrm{~m}, 1 \mathrm{H}), 3.41(\mathrm{~s}$, $2 \mathrm{H}), 1.97$ (s, $3 \mathrm{H}), 1.56$ (s, $6 \mathrm{H})$.

${ }^{13} \mathrm{C}$ NMR (150 MHz, $\mathrm{CD}_{3} \mathrm{OD}$ ): $\delta=172.4,160.3,150.7,138.2,125.3$, 123.7, 123.5, 121.4, 120.5, 119.3, 112.8, 105.4, 81.6, 49.1, 38.8, 26.8, 22.4 .

HRMS: $m / z[\mathrm{M}+\mathrm{H}]^{+}$calcd for $\mathrm{C}_{17} \mathrm{H}_{19} \mathrm{~N}_{2} \mathrm{O}_{3}$ : 299.1396; found: 299.1390 .

Preparation of Oxazoles 21 and 22 with MW Technique in the Presence of Propylphosphonic Anhydride (T3P ${ }^{\circledR}$ ); General Procedure

Ketoamide (19 or $\mathbf{2 0} ; 0.5 \mathrm{mmol}$ ), T3P ${ }^{\circledR}$ reagent (10 equiv, $2.98 \mathrm{~mL}, 50 \%$ EtOAc solution) and $\mathrm{CH}_{3} \mathrm{CN}(12 \mathrm{~mL})$ were measured into a vent-andreseal vessel and the reaction mixture was stirred at $100^{\circ} \mathrm{C}$ for $1 \mathrm{~h}$ under microwave irradiation. After cooling to r.t., the mixture was evaporated, the residue was taken up in $\mathrm{CH}_{2} \mathrm{Cl}_{2}(50 \mathrm{~mL})$ and extracted with sat. aq. $\mathrm{NaHCO}_{3}(2 \times 25 \mathrm{~mL})$ and water $(4 \times 25 \mathrm{~mL})$. The organic layer was dried $\left(\mathrm{MgSO}_{4}\right)$, filtered and evaporated to afford the appropriate product. Analytical samples were obtained by recrystallization from aqueous EtOH.

\section{Methyl [5-(1H-Indol-3-yl)-1,3-oxazol-2-yl]acetate (21)}

Yield: $126 \mathrm{mg}$ (98\%); brown crystals; $\mathrm{mp} 158-160{ }^{\circ} \mathrm{C}\left(\mathrm{EtOH}-\mathrm{H}_{2} \mathrm{O}\right)$. IR (KBr): 3170, 2899, 1746, 1637, 1251, 1169, 1006, $739 \mathrm{~cm}^{-1}$. ${ }^{1} \mathrm{H}$ NMR $\left(600 \mathrm{MHz}, \mathrm{CD}_{3} \mathrm{OD}\right): \delta=7.79(\mathrm{~d}, J=7.9 \mathrm{~Hz}, 1 \mathrm{H}), 7.63(\mathrm{~s}, 1 \mathrm{H})$, $7.43(\mathrm{~d}, J=8.0 \mathrm{~Hz}, 1 \mathrm{H}), 7.24(\mathrm{~s}, 1 \mathrm{H}), 7.20(\mathrm{t}, J=7.3 \mathrm{~Hz}, 1 \mathrm{H}), 7.15(\mathrm{t}, J=$ $7.5 \mathrm{~Hz}, 1 \mathrm{H}), 3.99$ (s, $1 \mathrm{H}), 3.98(\mathrm{~s}, 1 \mathrm{H}), 3.77(\mathrm{~s}, 3 \mathrm{H})$.

${ }^{13} \mathrm{C}$ NMR (150 MHz, $\left.\mathrm{CD}_{3} \mathrm{OD}\right): \delta=169.8,156.9,151.1,138.2,125.3$, $124.1,123.5,121.4,120.5,119.7,112.8,105.2,53.1,34.8$.

HRMS: $m / z[\mathrm{M}+\mathrm{H}]^{+}$calcd for $\mathrm{C}_{14} \mathrm{H}_{13} \mathrm{~N}_{2} \mathrm{O}_{3}$ : 257.0926; found: 257.0921 .

\section{3-[2-(2-Methylprop-1-en-1-yl)-1,3-oxazol-5-yl]-1H-indole (22,} labradorin 5)

Yield: $188 \mathrm{mg}(99 \%)$; brown crystals; mp $126-129^{\circ} \mathrm{C}\left(\right.$ EtOH- $\left.\mathrm{H}_{2} \mathrm{O}\right)$ (lit. ${ }^{7} \mathrm{mp} 128-131^{\circ} \mathrm{C}$ ).

IR (KBr): 3452, 3063, 1631, 1449, 1112, 1011, 978, $735 \mathrm{~cm}^{-1}$.

${ }^{1} \mathrm{H}$ NMR $\left(600 \mathrm{MHz}, \mathrm{CD}_{3} \mathrm{OD}\right): \delta=7.81(\mathrm{~d}, J=7.9 \mathrm{~Hz}, 1 \mathrm{H}), 7.62(\mathrm{~s}, 1 \mathrm{H})$, $7.44(\mathrm{~d}, J=8.0 \mathrm{~Hz}, 1 \mathrm{H}), 7.27(\mathrm{~s}, 1 \mathrm{H}), 7.20(\mathrm{t}, J=7.3 \mathrm{~Hz}, 1 \mathrm{H}), 7.16(\mathrm{t}, J=$ $7.4 \mathrm{~Hz}, 1 \mathrm{H}), 6.17(\mathrm{~s}, 1 \mathrm{H}), 2.27(\mathrm{~s}, 3 \mathrm{H}), 2.01(\mathrm{~s}, 3 \mathrm{H})$.

${ }^{13} \mathrm{C}$ NMR (150 MHz, $\left.\mathrm{CD}_{3} \mathrm{OD}\right): \delta=160.7,149.0,146.5,138.2,125.3$, 123.9, 123.5, 121.4, 120.5, 120.0, 112.8, 112.3, 105.5, 27.4, 20.7. 


\section{Preparation of Pimprinol Alkaloids 1-3; General Procedure} Acetoxy-protected oxazole (11, 16 or $\mathbf{2 7} ; 0.7 \mathrm{mmol})$ was dissolved in $\mathrm{MeOH}(28 \mathrm{~mL}$ ) and aq. $\mathrm{NaOH}$ (3.08 mmol in $0.5 \mathrm{~mL}$ water, 4.4 equiv) was added. The reaction mixture was stirred at r.t. until the reaction was complete, then the solvent was evaporated. The residue was taken up in a mixture of EtOAc $(50 \mathrm{~mL})$ and water $(50 \mathrm{~mL})$, and the phases were separated. The aqueous mixture was extracted with further EtOAc $(3 \times 30 \mathrm{~mL})$, and the combined organic phases were dried over $\mathrm{MgSO}_{4}$, filtered and evaporated. The products were purified by recrystallization from $\mathrm{MeCN}$.

\section{1-[5-(1H-Indol-3-yl)-1,3-oxazol-2-yl]ethanol [(rac)-1]}

Yield: $155 \mathrm{mg}$ (97\%); pale-yellow crystals; $\mathrm{mp} 157-160{ }^{\circ} \mathrm{C}(\mathrm{MeCN})$ (lit. $\left.{ }^{6} \mathrm{mp} 151-152{ }^{\circ} \mathrm{C}\right)$.

IR (KBr): 3221, 1638, 1445, 1338, 1241, 1084, 974, $751 \mathrm{~cm}^{-1}$. ${ }^{1} \mathrm{H}$ NMR $\left(600 \mathrm{MHz}, \mathrm{CD}_{3} \mathrm{OD}\right): \delta=7.82(\mathrm{~d}, J=7.9 \mathrm{~Hz}, 1 \mathrm{H}), 7.66(\mathrm{~s}, 1 \mathrm{H})$, $7.44(\mathrm{~d}, J=8.1 \mathrm{~Hz}, 1 \mathrm{H}), 7.22(\mathrm{~s}, 1 \mathrm{H}), 7.20(\mathrm{t}, J=7.3 \mathrm{~Hz}, 1 \mathrm{H}), 7.16(\mathrm{t}, J=$ $7.9 \mathrm{~Hz}, 1 \mathrm{H}), 4.96(\mathrm{q}, J=6.7 \mathrm{~Hz}, 1 \mathrm{H}), 1.63(\mathrm{~d}, J=6.7 \mathrm{~Hz}, 3 \mathrm{H})$.

${ }^{13} \mathrm{C}$ NMR $\left(150 \mathrm{MHz}, \mathrm{CD}_{3} \mathrm{OD}\right): \delta=165.1,150.4,138.2,125.3,124.0$, $123.5,121.4,120.5,119.2,112.8,105.3,64.2,21.5$.

(1R)-1-[5-(1H-Indol-3-yl)-1,3-oxazol-2-yl]ethanol ([(R)-1], pimprinol $A$ )

Yield: $157 \mathrm{mg}$ (98\%); $98 \%$ ee; pale-yellow crystals; mp $172-174{ }^{\circ} \mathrm{C}$ (MeCN) (lit. $\left.{ }^{6} \mathrm{mp} 151-152^{\circ} \mathrm{C}\right) ;[\alpha]_{\mathrm{D}}{ }^{27}+8.2(\mathrm{c} 1.0, \mathrm{MeOH})$.

IR (KBr): 3267, 1680, 1440, 1385, 1128, 1098, 971, $732 \mathrm{~cm}^{-1}$.

${ }^{1} \mathrm{H}$ NMR $\left(600 \mathrm{MHz}, \mathrm{CD}_{3} \mathrm{OD}\right): \delta=7.84-7.81(\mathrm{~m}, 1 \mathrm{H}), 7.65(\mathrm{~s}, 1 \mathrm{H})$, 7.46-7.43 (m, $1 \mathrm{H}), 7.22(\mathrm{~s}, 1 \mathrm{H}), 7.24-7.18(\mathrm{~m}, 1 \mathrm{H}), 7.17-7.14(\mathrm{~m}$, $1 \mathrm{H}), 4.96(\mathrm{q}, J=6.7 \mathrm{~Hz}, 1 \mathrm{H}), 1.63(\mathrm{~d}, J=6.7 \mathrm{~Hz}, 3 \mathrm{H})$.

${ }^{13} \mathrm{C}$ NMR $\left(150 \mathrm{MHz}, \mathrm{CD}_{3} \mathrm{OD}\right): \delta=165.1,150.4,138.2,125.3,124.0$, $123.5,121.4,120.5,119.2,112.8,105.3,64.2,21.5$.

(1S)-1-[5-(1H-Indol-3-yl)-1,3-oxazol-2-yl]ethanol [(S)-1]

Yield: $142 \mathrm{mg}$ (89\%); $98 \%$ ee; pale-yellow crystals; mp $177-179{ }^{\circ} \mathrm{C}$ $(\mathrm{MeCN}) ;[\alpha]_{\mathrm{D}}{ }^{28}-7.4$ (c $\left.1.0, \mathrm{MeOH}\right)$.

IR (KBr): 3266, 1681, 1440, 1376, 1128, 1098, 972, $732 \mathrm{~cm}^{-1}$.

${ }^{1} \mathrm{H}$ NMR $\left(600 \mathrm{MHz}, \mathrm{CD}_{3} \mathrm{OD}\right): \delta=7.84-7.81(\mathrm{~m}, 1 \mathrm{H}), 7.65(\mathrm{~s}, 1 \mathrm{H})$, 7.46-7.42 (m, $1 \mathrm{H}), 7.22(\mathrm{~s}, 1 \mathrm{H}), 7.24-7.18(\mathrm{~m}, 1 \mathrm{H}), 7.17-7.14(\mathrm{~m}$, $1 \mathrm{H}), 4.96(\mathrm{q}, J=6.7 \mathrm{~Hz}, 1 \mathrm{H}), 1.63(\mathrm{~d}, J=6.7 \mathrm{~Hz}, 3 \mathrm{H})$.

${ }^{13} \mathrm{C}$ NMR $\left(150 \mathrm{MHz}, \mathrm{CD}_{3} \mathrm{OD}\right): \delta=165.1,150.4,138.2,125.3,124.0$, $123.5,121.4,120.5,119.2,112.8,105.3,64.2,21.5$.

HRMS: $m / z[\mathrm{M}+\mathrm{H}]^{+}$calcd for $\mathrm{C}_{13} \mathrm{H}_{13} \mathrm{~N}_{2} \mathrm{O}_{2}$ : 229.0977; found: 229.0972 .

\section{1-[5-(1H-Indol-3-yl)-1,3-oxazol-2-yl]-2-methylpropan-2-ol (2,} pimprinol B $)^{6}$

Yield: $149 \mathrm{mg}$ (83\%); colorless crystals; $\mathrm{mp} 149-150{ }^{\circ} \mathrm{C}(\mathrm{MeCN})$. IR (KBr): 3222, 1636, 1441, 1338, 1257, 1107, 971, $741 \mathrm{~cm}^{-1}$. ${ }^{1} \mathrm{H}$ NMR $\left(600 \mathrm{MHz}, \mathrm{CD}_{3} \mathrm{OD}\right): \delta=7.81(\mathrm{~d}, J=7.7 \mathrm{~Hz}, 1 \mathrm{H}), 7.61(\mathrm{~s}, 1 \mathrm{H})$, $7.43(\mathrm{~d}, J=8.0 \mathrm{~Hz}, 1 \mathrm{H}), 7.24-7.11(\mathrm{~m}, 3 \mathrm{H}), 3.00(\mathrm{~s}, 1 \mathrm{H}), 1.34(\mathrm{~s}, 6 \mathrm{H})$.

${ }^{13} \mathrm{C}$ NMR $\left(150 \mathrm{MHz}, \mathrm{CD}_{3} \mathrm{OD}\right): \delta=164.5,150.4,138.2,125.3,124.0$, $123.5,121.4,120.5,119.1,112.8,105.3,69.7,29.6,10.1$.

\section{1-[5-(1-Indol-3-yl)-1,3-oxazol-2-yl]propan-1-ol [(rac)-3]}

Yield: $161 \mathrm{mg}$ (95\%); colorless crystals; $\mathrm{mp} 159-160{ }^{\circ} \mathrm{C}(\mathrm{MeCN})$. IR (KBr): 3222, 1636, 1441, 1338, 1257, 1107, 971, $741 \mathrm{~cm}^{-1}$.
${ }^{1} \mathrm{H}$ NMR $\left(600 \mathrm{MHz}, \mathrm{CD}_{3} \mathrm{OD}\right): \delta=7.82(\mathrm{~d}, J=7.8 \mathrm{~Hz}, 1 \mathrm{H}), 7.65(\mathrm{~s}, 1 \mathrm{H})$, $7.44(\mathrm{~d}, J=8.1 \mathrm{~Hz}, 1 \mathrm{H}), 7.23(\mathrm{~s}, 1 \mathrm{H}), 7.20(\mathrm{t}, J=7.2 \mathrm{~Hz}, 1 \mathrm{H}), 7.16(\mathrm{t}, J=$ $7.8 \mathrm{~Hz}, 1 \mathrm{H}), 4.70(\mathrm{t}, J=6.9 \mathrm{~Hz}, 1 \mathrm{H}), 2.07-1.93(\mathrm{~m}, 2 \mathrm{H}), 1.01(\mathrm{t}, J=$ $7.4 \mathrm{~Hz}, 3 \mathrm{H}) \mathrm{ppm}$.

${ }^{13} \mathrm{C}$ NMR $\left(150 \mathrm{MHz}, \mathrm{CD}_{3} \mathrm{OD}\right): \delta=164.5,150.4,138.2,125.3,124.0$, $123.5,121.4,120.5,119.1,112.8,105.3,69.7,29.6,10.1$.

HRMS: $m / z[\mathrm{M}+\mathrm{H}]^{+}$calcd for $\mathrm{C}_{14} \mathrm{H}_{15} \mathrm{~N}_{2} \mathrm{O}_{2}$ : 243.1134; found: 243.1128 .

(1R)-1-[5-(1-Indol-3-yl)-1,3-oxazol-2-yl]propan-1-ol ([(R)-3], pimprinol C) ${ }^{6}$

Yield: $158 \mathrm{mg}$ (93\%); $98 \%$ ee; colorless crystals; mp $143-145{ }^{\circ} \mathrm{C}$ $(\mathrm{MeCN}) ;[\alpha]_{D}^{27}+12.8(\mathrm{c} 1.0, \mathrm{MeOH})$.

IR (KBr): 3285, 1634, 1587, 1438, 1258, 1125, 966, $732 \mathrm{~cm}^{-1}$.

${ }^{1} \mathrm{H}$ NMR $\left(600 \mathrm{MHz}, \mathrm{CD}_{3} \mathrm{OD}\right): \delta=7.82(\mathrm{~d}, J=7.9 \mathrm{~Hz}, 1 \mathrm{H}), 7.65(\mathrm{~s}, 1 \mathrm{H})$ $7.44(\mathrm{~d}, J=8.1 \mathrm{~Hz}, 1 \mathrm{H}), 7.23(\mathrm{~s}, 1 \mathrm{H}), 7.26-7.18(\mathrm{~m}, 1 \mathrm{H}), 7.17-7.13(\mathrm{~m}$, $1 \mathrm{H}), 4.70(\mathrm{t}, J=7.0 \mathrm{~Hz}, 1 \mathrm{H}), 2.07-1.93(\mathrm{~m}, 2 \mathrm{H}), 1.01(\mathrm{t}, J=7.4 \mathrm{~Hz}$, $3 \mathrm{H})$.

${ }^{13} \mathrm{C}$ NMR (150 MHz, $\left.\mathrm{CD}_{3} \mathrm{OD}\right): \delta=164.5,150.4,138.2,125.3,124.0$, $123.5,121.4,120.5,119.1,112.8,105.4,69.7,29.6,10.1$.

\section{(1S)-1-[5-(1-Indol-3-yl)-1,3-oxazol-2-yl]propan-1-ol [(S)-3]}

Yield: $162 \mathrm{mg}$ (96\%); $98 \%$ ee; pale-yellow crystals; mp $144-146{ }^{\circ} \mathrm{C}$ $(\mathrm{MeCN}) ;[\alpha]_{D}^{27}-12.2$ (c 1.0, MeOH).

IR (KBr): 3259, 1634, 1615, 1439, 1127, 977, 800, $732 \mathrm{~cm}^{-1}$.

${ }^{1} \mathrm{H}$ NMR $\left(600 \mathrm{MHz}, \mathrm{CD}_{3} \mathrm{OD}\right): \delta=7.82(\mathrm{~d}, J=7.9 \mathrm{~Hz}, 1 \mathrm{H}), 7.65(\mathrm{~s}, 1 \mathrm{H})$, $7.44(\mathrm{~d}, J=8.1 \mathrm{~Hz}, 1 \mathrm{H}), 7.23(\mathrm{~s}, 1 \mathrm{H}), 7.26-7.18(\mathrm{~m}, 1 \mathrm{H}), 7.17-7.13(\mathrm{~m}$, $1 \mathrm{H}), 4.70(\mathrm{t}, J=7.1 \mathrm{~Hz}, 1 \mathrm{H}), 2.07-1.93(\mathrm{~m}, 2 \mathrm{H}), 1.01(\mathrm{t}, J=7.4 \mathrm{~Hz}$, $3 \mathrm{H})$.

${ }^{13} \mathrm{C}$ NMR $\left(150 \mathrm{MHz}, \mathrm{CD}_{3} \mathrm{OD}\right): \delta=164.5,150.4,138.2,125.3,124.0$, 123.5, 121.4, 120.5, 119.1, 112.8, 105.4, 69.7, 29.6, 10.1 .

HRMS: $m / z[\mathrm{M}+\mathrm{H}]^{+}$calcd for $\mathrm{C}_{14} \mathrm{H}_{15} \mathrm{~N}_{2} \mathrm{O}_{2}: 243.1134$; found: 243.1128 .

\section{Supporting Information}

Supporting information for this article is available online at https://doi.org/10.1055/s-0039-1690336.

\section{References}

(1) Jacobs, H. M.; Burke, B. A. Alkaloids, Vol. 35; Rossi, A., Ed.; Academic Press: San Diego, 1989, 259-310.

(2) Davyt, D.; Serra, G. Mar. Drugs 2010, 8, 2755.

(3) Swellmeen, L. Pharma Chem. 2016, 8, 269.

(4) Joshi, S.; Bisht, A. S.; Juyal, D. Pharma Innovation 2017, 6, 109.

(5) Kakkar, S.; Narasimhan, B. BMC Chem. 2019, 13, 16.

(6) Raju, R.; Gromyko, O.; Fedorenko, V.; Luzhetskyy, A.; Müller, R. Tetrahedron Lett. 2012, 53, 3009.

(7) Xiang, J.; Wang, J.; Wang, M.; Meng, X.; Wu, A. Tetrahedron 2014, 70, 7470 .

(8) Kolasa, T.; Miller, M. J. J. Org. Chem. 1987, 52, 4978.

(9) Testa, E.; Fontanella, L.; Mariani, L. Justus Liebigs Ann. Chem. 1962, 660, 135

(10) Leśniak, S.; Rachwalski, M.; Jarzyński, S.; Obijalska, E. Tetrahedron: Asymmetry 2013, 24, 1336.

(11) Szabó, T.; Dancsó, A.; Ábrányi-Balogh, P.; Volk, B.; Milen, M. Tetrahedron Lett. 2019, 60, 1353. 
(12) Nicolaou, K. C.; Hao, J.; Reddy, M. V.; Rao, P. B.; Rassias, G.; Snyder, S. A.; Huang, X.; Chen, D. Y.-K.; Brenzovich, W. E.; Giuseppone, N.; Giannakakou, P.; O’Brate, A. J. Am. Chem. Soc. 2004, 126, 12897.

(13) Clayden, J.; Greeves, N.; Warren, S.; Wothers, P. Organic Chemistry, 1st ed; Oxford University Press: Oxford, 2001, 1223.

(14) Crombie, L.; Jones, R. C. F.; Palmer, C. J. J. Chem. Soc., Perkin Trans. 1 1987, 333.

(15) Oruganti, S.; Sen, S.; Dahanukar, V. H.; Ganorkar, R.; Chakka, R. PCT Int. Appl. WO 2016108206, 2016; Chem. Abstr. 2016, 165, 176459.

(16) Claffey, M. M.; Helal, C. J.; Verhoest, P. R. PCT Int. Appl. WO 2010084438, 2010; Chem. Abstr. 2010, 153, 260362.

(17) Suess, B.; Brockhoff, A.; Meyerhof, W.; Hofmann, T. J. Agric. Food Chem. 2018, 66, 2301.

(18) Moon, H. R.; Kim, S. Y.; Lim, J. W.; Kim, J. N. Bull. Korean Chem. Soc. 2015, 36, 2773.
(19) Morisaki, M.; Rubio-Lightbourn, J.; Ikekawa, N. Chem. Pharm. Bull. 1973, 21, 457.

(20) Almansour, A. I.; Arumugam, N.; Kumar, R. S.; Soliman, S. M. Altaf M.; Ghabbour, H. A. Molecules 2016, 21, 724.

(21) Chudasama, V.; Ahern, J. M.; Fitzmaurice, R. J.; Caddick, S. Tetrahedron Lett. 2011, 52, 1067.

(22) Nomura, T.; Fukai, T.; Sato, E.; Fukushima, K. Heterocycles 1981 16, 983.

(23) Fedeor, L. R. J. Am. Chem. Soc. 1969, 91, 913.

(24) Nemet, I.; Varga-Defterdarovic, L. Bioorg. Med. Chem. 2008, 16, 4551.

(25) Muratore, M. E.; Shi, L.; Pilling, A. W.; Storer, R. I.; Dixon, D. J. Chem. Commun. 2012, 48, 6351.

(26) Broberg, A.; Bjerketorp, J.; Andersson, P.; Sahlberg, C.; Levenfors, J. J. Molecules 2017, 22, 1072.

(27) Miyake, F.; Hashimoto, M.; Tonsiengsom, S.; Yakushijin, K.; Horne, D. A. Tetrahedron 2010, 66, 4888. 